\title{
NONLOCAL VARIATIONAL PROBLEMS ARISING IN LONG WAVE PROPAGATION
}

\author{
ORLANDO LOPES ${ }^{1}$
}

\begin{abstract}
In this paper we study the existence of minimizer for certain constrained variational problems given by functionals with nonlocal terms. This type of functionals are first integrals of evolution equations describing long wave propagation and the existence of minimizer gives the existence and the stability of traveling waves for these equations. Due to loss of compactness, the major problem is to prevent dichotomy of minimizing sequences. Our approach is an alternative to the concentrationcompactness method and it allows us to deal with some functionals for which the verification of the strict subadditivity seems to be difficult.
\end{abstract}

AMS Subject Classification. 35J20, 49J10.

Received January 20, 2000. Revised August, 2000.

\section{INTRODUCTION}

In this paper we consider the following problem:

$$
\text { (P) Minimize } V(\mathbf{u}) \text { subject to } I(\mathbf{u})=\lambda>0 \text {, }
$$

where $V(\mathbf{u})$ and $I(\mathbf{u})$ will be translation invariant functionals defined on the Hilbert space $H_{K}^{s}\left(R^{N}\right), s \geq 1 / 2$, of the vector-valued functions $\mathbf{u}: R^{N} \rightarrow R^{K}, \mathbf{u}(x)=\left(u_{1}(x), \cdots, u_{K}(x)\right)$ such that

$$
|\mathbf{u}|_{s}^{2} \widehat{=} \int_{R^{N}}\left(1+|\xi|^{2 s}\right)|\hat{\mathbf{u}}(\xi)|^{2} \mathrm{~d} \xi=\int_{R^{N}}\left(1+|\xi|^{2 s}\right)\left(\sum_{i=1}^{K}\left|\hat{u}_{i}(\xi)\right|^{2}\right) \mathrm{d} \xi<\infty
$$

where, as usual, $\hat{u}(\xi)$ denotes the Fourier transform of $u(x)$.

A typical example is

and

$$
V(\mathbf{u})=\frac{1}{2} \int_{R^{N}} m(\xi)|\hat{\mathbf{u}}(\xi)|^{2} \mathrm{~d} \xi+\int_{R^{N}} F(\mathbf{u}(x)) \mathrm{d} x
$$

$$
I(\mathbf{u})=\int_{R^{N}} G(\mathbf{u}(x)) \mathrm{d} x
$$

$F(\mathbf{u})$ and $G(\mathbf{u})$ being real valued functions defined on the space $R^{K}$ and $m(\xi)$ is a symbol defined for $\xi \in R^{N}$.

Keywords and phrases: Nonlocal variational problems, stability of traveling waves.

1 Departamento de Matematica-IMECC-UNICAMP- CP 6065, Campinas SP 13083-970, Brasil; e-mail: 1opes@ime.unicamp.br 
Our main motivation is to study such problems in connection with evolution equations. For instance, if

$$
\dot{\mathbf{u}}(t)=K(\mathbf{u}(t))
$$

is an evolution equation in a Hilbert space $X$ and $V(\mathbf{u})$ and $I(\mathbf{u})$ are conserved quantities for (1.1), then for any real number $\alpha, V(\mathbf{u})+\alpha I(\mathbf{u})$ is also a conserved quantity and then, according to a result of Lax [17], the set of the critical points of $V(\mathbf{u})+\alpha I(\mathbf{u})$, that is, the set of the elements $\mathbf{u} \in X$ such that $V^{\prime}(\mathbf{u})+\alpha I^{\prime}(\mathbf{u})=0$, is also invariant under (1.1). Such critical points give rise to special solutions of equation (1.1) (traveling waves, standing waves, bound states). Moreover, if such critical points are minimizers of problem (P), then such special solutions are stable with respect to (1.1) (see [10]). In some cases uniqueness of weak solutions of the Cauchy problem for the evolution equation (1.1) is not known and, because of that, the stability of the waves is proved for positive time only.

So, in the applications, for a given value of $\lambda$, the set of the minimizers of problem $(\mathbf{P})$ is stable with respect to (1.1) and it consists of special solutions. A more difficult question is to describe this set of minimizers. For instance, is it made of a finite number of orbits? (see comments about this point in the introduction of [2]). The answer to this question depends on knowing the multiplicity of the zero eigenvalue of the linearized operator $V^{\prime \prime}(\mathbf{u})+\alpha I^{\prime \prime}(\mathbf{u})$ and, in general, this seems to be a difficult problem.

A different attitude is to assume that there is a smooth curve of critical points $\mathbf{u}(c)$ parametrized by the speed $c$ and to study its stability or instability by looking at the function $V(\mathbf{u}(c))+c I(\mathbf{u}(c)$ ) (see [14] and [30], among many others) (instability cannot be proved by the minimization method of [10]). In this approach, we also have to know the multiplicity of the zero eigenvalue of the linearized operator (see [31], for instance).

If $N \geq 2$ and the symbol $m(\xi)$ is equal to some $m_{0}(|\xi|)$ then the functionals $V(\mathbf{u})$ and $I(\mathbf{u})$ are also invariant under rotations and then we can minimize $V(\mathbf{u})$ under $I(\mathbf{u})=\lambda$ in the space $H_{K \text {,rad }}^{s}\left(R^{N}\right)$ of the functions that are radially symmetric. For instance, if $s=1$, due to the compact imbedding of $H_{\text {rad }}^{1}\left(R^{N}\right)$ into $L^{p}\left(R^{N}\right)$ for $2<p<2^{*}=\frac{2 N}{N-2}$, that minimization problem can be solved easily. But if we want to apply that type of result to conclude stability of waves, then we would get stability just with respect to perturbations which are radially symmetric. In other words, if we want to get stability of waves with respect to a large class of perturbations, then we cannot restrict the minimization problem $(\mathbf{P})$ to the class of radially symmetric functions. However, for some types of variational problems, the radial symmetry of the minimizers can be proved [19,22].

Due to the invariance of the problem $(\mathbf{P})$ with respect to translation in the space variable, the best we can ask is if minimizing sequences are precompact except for translations in the space variable and the main difficulty is the possibility of having dichotomy of a minimizing sequence.

This class of problems has been studied by Lions in a series of papers ([20] and [21] among many others) and, for instance, if $G(\mathbf{u})=|\mathbf{u}|^{2}$, it has been proved that preventing dichotomy is equivalent to verify the strict inequality

$$
V(\lambda)<V(\mu)+V(\lambda-\mu), \quad 0<\mu<\lambda
$$

where $V(\lambda)$ is the infimum of $V(\mathbf{u})$ on the admissible set $\{\mathbf{u}: I(\mathbf{u})=\lambda\}$. This strict inequality has been verified in many examples and that method, called concentration-compactness, has been widely used.

In [23-25] and [26] we have offered a different approach for that type of problem in the case of one constraint (concentration-compactness works also in the case of many constraints [9,21]) and, according to that, dichotomy is prevented provided we can show that any nontrivial solution of the Euler-Lagrange

$$
V^{\prime}(\mathbf{u})+\alpha I^{\prime}(\mathbf{u})=0
$$

which is the weak limit of a minimizing sequence of problem $(\mathbf{P})$ has Morse index at least one (assumption $\mathbf{H}_{\mathbf{5}}$ of Th. 2.1 in this paper). Since it can be shown very easily that the Morse index of such a limit cannot be larger than one, that implies that the Morse index has to be exactly one. 
As we have done in simpler and more concrete situations, the main idea is to show that under assumption $\mathbf{H}_{\mathbf{5}}$ of Theorem 2.1, dichotomy violates a second derivative condition for minimizing sequences. In order to carry out this program we use a lemma of Lions which is related to the so called Lieb's lemma.

In the case of two constraints and under assumption $\mathbf{H}_{5}$, all we can show is that a minimizing sequence of problem (P) cannot "tricotomize", but it is not clear what are the implications of this fact. The multiconstrained problem also arises naturally in the study of the existence and stability of traveling waves for a fourth order wave equation [18] and of the stability of double waves for the integrable KdV [27]. This last case shows that dichotomy may indeed occur (if we believe that the double wave is actually is a global minimizer of the corresponding variational problem; all we know is that it is a local minimizer).

So, in the case of one constraint, we can choose between verifying (1.2) or assumption $\mathbf{H}_{\mathbf{5}}$ of Theorem 2.1.

In several examples for which inequality (1.2) has been proved, we were also been able to verify assumption $\mathbf{H}_{\mathbf{5}}$ under similar conditions on the functions appearing in the functional (in general, we require more derivatives). On the other hand, there are examples for which we can verify assumption $\mathbf{H}_{\mathbf{5}}$ and, apparently, it would be difficult to prove the strict inequality (1.2). Of course we cannot say that it would be impossible because (1.2) is also a necessary condition for preventing dichotomy.

In this paper we use our alternative approach to study a class of functionals with a nonlocal term that arise in the theory of long wave propagation and that has been studied in [2-4] and [31]. More general models have been studied in [28] and the conservative system

$$
\frac{\partial \mathbf{u}}{\partial t}+\sum_{i=1}^{N} \frac{\partial}{\partial x_{i}}[\operatorname{grad} F(\mathbf{u})+\mathbf{L u}]=0
$$

has two first integrals $V(\mathbf{u})$ and $I(\mathbf{u})$ of the type we consider in this paper.

As an application of our method we will show the existence of a stable set of traveling waves of the modified generalized intermediate long wave equation

$$
u_{t}+\left(f(u(x))_{x}-\beta_{1} M_{1} u_{x}-\beta_{2} M_{2} u_{x}=0\right.
$$

under minimal assumptions on the nonlinearity $f(u)$, where $\beta_{i}>0, i=1,2, M_{i}$ is the Fourier multiplier defined by

$$
\left(\widehat{M}_{i} w\right)(\xi)=m_{i}(\xi) \hat{w}(\xi), \quad m_{i}(\xi)=\xi \operatorname{coth}\left(\xi H_{i}\right)-\frac{1}{H_{i}}
$$

for $i=1,2, H_{i}$ a real number. The modified Benjamin-Ono equation can be treated in a similar way.

From the point of view of our theory, equation (1.4) is special because a sort of maximum principle maximum is available for it and, because of that, we are able to show that assumption $\mathbf{H}_{\mathbf{5}}$ is satisfied for a very large class of nonlinearities $f(u)$ and this will allow us to solve the corresponding minimization problem.

In the case of local functionals, we have developed our approach in previous papers and here we just indicate some applications of that case to a KdV system, a Schrodinger system, the generalized Zakharov-Kuznetsov equation [8] and the "BBM" version of it.

In order to avoid repetition of arguments in future applications and to cover some existing results, the method will be presented as abstractly as possible. In the last section we make specific applications of our theory. The main result of the abstract part is to show that, under asssumption $\mathbf{H}_{\mathbf{5}}$, dichotomy is prevented and the crucial argument is to show that, in presence of assumption $\mathbf{H}_{\mathbf{5}}$, dichotomy violates a second derivative condition for minimizing sequences. We also prove an abstract stability result (in the spirit of [10]) of the set where the minimum of problem $(\mathbf{P})$ is achieved.

\section{ThE ABSTRACT THEORY}

Notation. We will use boldface letters $\mathbf{u}, \mathbf{h}$ to indicate either that $\mathbf{u}, \mathbf{h}$ are vectors in $R^{K}$ or that they are $R^{K}$-vector valued functions. $L_{K}^{p}\left(R^{N}\right)$ is the space of the $R^{K}$-valued functions defined on $R^{N}$ whose components 
are in $L^{p}\left(R^{N}\right) . H_{K}^{s}\left(R^{N}\right)$ is the space of the functions $\mathbf{u}: R^{N} \rightarrow R^{K}, \mathbf{u}(x)=\left(u_{1}(x), \cdots, u_{K}(x)\right)$ such that

$$
|\mathbf{u}|_{s}^{2} \widehat{=} \int_{R^{N}}\left(1+|\xi|^{2 s}\right)|\hat{\mathbf{u}}(\xi)|^{2} \mathrm{~d} \xi=\int_{R^{N}}\left(1+|\xi|^{2 s}\right)\left(\sum_{i=1}^{K}\left|\hat{u}_{i}(\xi)\right|^{2}\right) \mathrm{d} \xi<\infty,
$$

where $\hat{u}(\xi)$ denotes the Fourier transform of $u(x)$; if $K=1$ it will be omitted. The scalar product and the norm in $H_{K}^{s}\left(R^{N}\right)$ will be denoted by $\langle,\rangle_{s}$ and ||$_{s}$, respectively, and the scalar product and norm in $R^{K}$ simply by $\langle$, and ||, respectively.

We consider the following problem;

$$
\text { (P) Minimize } V(\mathbf{u}) \quad \text { subject to } \quad I(\mathbf{u})=\lambda>0
$$

where $V(\mathbf{u})$ and $I(\mathbf{u})$ are functionals defined on the space $H_{K}^{s}\left(R^{N}\right), s>0$, satisfying the following assumptions: $\left.\mathbf{H}_{1}\right) V, I: H_{K}^{s}\left(R^{N}\right) \rightarrow R$ are translation invariant $C^{2}$ functionals whose first and second derivatives are uniformly continuous on bounded sets, $V(0)=0$ and $I(0)=0$;

$\left.\mathbf{H}_{\mathbf{2}}\right)$ if $\mathbf{u}_{n} \in H_{K}^{s}\left(R^{N}\right)$ is a sequence converging weakly in $H_{K}^{s}\left(R^{N}\right)$ to $\mathbf{u}$ and $c_{n} \in R^{N}$ is such that $\left|c_{n}\right|$ tends to $\infty$ then for any $\mathbf{h}, \mathbf{k} \in H_{K}^{s}\left(R^{N}\right)$,

$$
I^{\prime}\left(\mathbf{u}_{n}\right)(\mathbf{h}), I^{\prime \prime}\left(\mathbf{u}_{n}\right)(\mathbf{h}, \mathbf{h}), V^{\prime}\left(\mathbf{u}_{n}\right)(\mathbf{h}) \text { and } V^{\prime \prime}\left(\mathbf{u}_{n}\right)(\mathbf{h}, \mathbf{h})
$$

converge to

respectively, and

$$
I^{\prime}(\mathbf{u})(\mathbf{h}), I^{\prime}(\mathbf{u})(\mathbf{h}, \mathbf{h}), V^{\prime}(\mathbf{u})(\mathbf{h}) \text { and } V^{\prime \prime}(\mathbf{u})(\mathbf{h}, \mathbf{h})
$$

$$
I^{\prime \prime}\left(\mathbf{u}_{n}\right)\left(\mathbf{h}, \mathbf{k}_{n}\right) \text { and } V^{\prime \prime}\left(\mathbf{u}_{n}\right)\left(\mathbf{h}, \mathbf{k}_{n}\right)
$$

converge to zero, where $\mathbf{k}_{n}(x)=\mathbf{k}\left(x+c_{n}\right)$;

$\mathbf{H}_{3}$ ) the admissible set $\left\{\mathbf{u} \in H_{K}^{s}\left(R^{N}\right): I(\mathbf{u})=\lambda\right\}$ is not empty and $I^{\prime}(\mathbf{u}) \neq 0$ for $\mathbf{u} \neq 0$ (a manifold condition);

$\left.\mathbf{H}_{4}\right) V$ is bounded below on admissible set $\left\{\mathbf{u} \in H_{K}^{s}\left(R^{N}\right): I(\mathbf{u})=\lambda\right\}$ and minimizing sequences are bounded in $H_{K}^{s}\left(R^{N}\right)$

$\left.\mathbf{H}_{\mathbf{5}}\right)$ if $\mathbf{u} \neq 0$ is the weak limit in $H_{K}^{s}\left(R^{N}\right)$ of a minimizing sequence for problem $(\mathbf{P})$ satisfying the EulerLagrange equation

$$
W^{\prime}(\mathbf{u})=V^{\prime}(\mathbf{u})+\alpha I^{\prime}(\mathbf{u})=0
$$

where $W(\mathbf{u}) \widehat{=} V(\mathbf{u})+\alpha I(\mathbf{u})$, then there is an element $\mathbf{h} \in H_{K}^{s}\left(R^{N}\right)$ such that

$$
W^{\prime \prime}(\mathbf{u})(\mathbf{h}, \mathbf{h})=V^{\prime \prime}(\mathbf{u})(\mathbf{h}, \mathbf{h})+\alpha I^{\prime \prime}(\mathbf{u})(\mathbf{h}, \mathbf{h})<0 .
$$

\section{Remarks.}

1) In assumption $\mathbf{H}_{\mathbf{5}}$, the fact that $\mathbf{u}$ solves an Euler equation is not an assumption (later we will show that any weak limit of a minimizing sequence satisfy an Euler equation like (2.1) as it was itself a minimizer); the assumption is the existence of an element $\mathbf{h}$ satisfying (2.2).

2) In problem $(\mathbf{P})$ we take $\lambda>0$ just for simplicity. The case $\lambda=0$ arises naturally in some problems when the space dimension $N$ is two and it could also be considered.

Our main abstract result is the following:

Theorem 2.1. Under assumptions $\mathbf{H}_{\mathbf{1}}$ to $\mathbf{H}_{\mathbf{5}}$, if $\mathbf{u}_{n}$ is a minimizing sequence for problem $(\mathbf{P})$ and $\mathbf{u}_{n}$ converges weakly in $H_{K}^{s}\left(R^{N}\right)$ to some $\mathbf{u} \neq 0$, then $\mathbf{u}_{n}$ converges to $\mathbf{u}$ strongly in $L_{K}^{p}\left(R^{N}\right), 2<p<\frac{2 N}{N-2 s}(2<p \leq+\infty$ if $N<2 s)$. 
Moreover, there are sequences of real numbers $\alpha_{n}$ and $\gamma_{n}$ and a sequence $\overline{\mathbf{h}}_{n}$ of elements of $H_{K}^{s}\left(R^{N}\right)$ such that:

i) $\alpha_{n}$ is bounded and $\gamma_{n}$ tends to zero;

ii) $\left|\overline{\mathbf{h}}_{n}\right|_{s}=1$;

iii) the following approximate Euler-Lagrange equation is satisfied:

$$
V^{\prime}\left(\mathbf{u}_{n}\right) \mathbf{h}+\alpha_{n} I^{\prime}\left(\mathbf{u}_{n}\right) \mathbf{h}+\gamma_{n}\left\langle\overline{\mathbf{h}}_{n}, \mathbf{h}\right\rangle_{s}=0 \text { for any } \mathbf{h} \in H_{K}^{s}\left(R^{N}\right) .
$$

Furthermore, u satisfies the exact Euler-Lagrange equation

$$
V^{\prime}(\mathbf{u})+\alpha I^{\prime}(\mathbf{u})=0
$$

for some real constant $\alpha$.

As we have already pointed out, the main idea of the proof of Theorem 2.1 is to show that, in the presence of assumption $\mathbf{H}_{\mathbf{5}}$, dichotomy violates a local condition of the second derivative of a minimizing sequence. All the rest is an Elementary Calculus proof.

Before starting the proof of Theorem 2.1 itself, we state and prove a few lemmata that will be useful. In all of them we suppose that assumptions $\mathbf{H}_{\mathbf{1}}$ to $\mathbf{H}_{\mathbf{5}}$ are satisfied.

The first is version for fractional spaces of a result of Lions ([20], Part II) which is related to the so called Lieb's lemma [7] and the second is a variant of the first. We start by recalling the definition and some properties of fractional spaces of real valued functions (see [29], for details).

The Lebesgue space $H^{s, p}\left(R^{N}\right), 1 \leq p<\infty$ is the set of the functions $u: R^{N} \rightarrow R$ such that $|u|_{s, p, R^{N}}=$ $\left|\mathcal{F}^{-1}\left\{\left(1+|\xi|^{2}\right)^{s / 2}(\mathcal{F} u)(\xi)\right\}\right|_{p}<\infty$, where $\mathcal{F}$ denotes Fourier transform. If $s=m+\sigma$ where $m$ is an integer and $0<\sigma<1$ then the Sobolev space $W^{s, p}\left(R^{N}\right)$ is the set of the functions $u$ such that

$$
\|u\|_{s, p}=\left[\|u\|_{m, p}^{p}+\sum_{|\alpha|=m} \int_{R^{N}} \int_{R^{N}} \frac{\left|D^{\alpha} u(x)-D^{\alpha} u(y)\right|^{p}}{|x-y|^{N+\sigma p}} \mathrm{~d} x d y\right]^{1 / p}<\infty .
$$

If $\Omega$ is a bounded $C^{\infty}$ domain of $R^{N}$ then $H^{s, p}(\Omega)$ is defined by restriction of elements of $H^{s, p}\left(R^{N}\right)$ and $|u|_{s, p, \Omega}=\inf |v|_{s, p, R^{N}}$ where the infimum is taken over the functions $v$ that coincide with $u$ in $\Omega$.

If $S\left(R^{N}\right)$ denotes the set of the rapidly decreasing infinitely differentiable functions defined on $R^{N}$ and $t \geq 0$, then $C^{t}\left(R^{N}\right)$ denotes the completion of $S\left(R^{N}\right)$ under the $C^{t}$ norm if $t$ is an integer or under the Holder norm if $t$ is not an integer.

Whenever necessary, we use the subscript $K$ to denote $R^{K}$-vector value functions. Sometimes, when $p=2$ it will omitted. For further reference we collect some important facts about the spaces defined above. Proofs may be found in [29] at the indicated section.

Lemma 2.2. The following properties hold:

1) $H^{s, 2}\left(R^{N}\right)=W^{s, 2}\left(R^{N}\right)$ (Sect. 2.3.3);

2) $H^{s, p}\left(R^{N}\right)=W^{s, p}\left(R^{N}\right)$ if $1<p<\infty$ and $s$ is an integer (Sect. 2.3.3);

3) if $1<p<\infty$ and $u \in H^{s, p}\left(R^{N}\right)$ then $\frac{\partial u}{\partial x_{i}}$ belongs to $H^{s-1, p}\left(R^{N}\right)$ (because $\frac{\xi_{i}}{\left(1+|\xi|^{2}\right)^{1 / 2}}$ is a M multiplier, $1<p<\infty)($ Sect. 2.3);

4) if either $A=R^{N}$ or $A=\Omega$ then

$$
\begin{aligned}
& {\left[H^{s_{1}, p_{1}}(A), H^{s_{2}, p_{2}}(A)\right]_{a}=H^{s, p}(A) \quad 1<p_{1}, p_{2}<\infty, \quad 0<a<1} \\
& s=(1-a) s_{1}+a s_{2} \quad \frac{1}{p}=\frac{1-a}{p_{1}}+\frac{a}{p_{2}} ;
\end{aligned}
$$

(Sect. 2.4.2 for $A=R^{N}$ and Sect. 4.3.1 for $A=\Omega$ ); 
5) if either $A=R^{N}$ or $A=\Omega$ then $H^{s, r}(A)$ is continuously imbedded in $L^{p}(A)$ if $1<r \leq p<\infty$ and $\frac{1}{p} \geq \frac{1}{r}-\frac{s}{N}$. Moreover, $H^{s, r}(A)$ is continuously imbedded in $L^{\infty}(A)$ if $\frac{1}{r}-\frac{s}{N}<0$ (Sect. 2.8.1 for $A=R^{N}$ and Sect. 4.6.1 for $A=\Omega$ );

6) $H^{s, r}(\Omega)$ is compactly imbedded in $L^{q}(\Omega)$ if $1<r \leq q \leq \infty$ and $\frac{1}{q}>\frac{1}{r}-\frac{s}{N}$ (Sect. 4.10.2);

7) $H^{t+N / p, p}\left(R^{N}\right)$ is continuously imbedded in $C^{t}\left(R^{N}\right), 1<p<\infty, 0<t \neq$ integer (Sect. 2.8.1);

8) for $2 \leq p<\infty$ and $\epsilon>0, H^{s, p}\left(R^{N}\right) \subset W^{s, p} \subset H^{s-\epsilon, p}$ (Sect. 2.3.3).

Lemma 2.3. Let $\mathbf{u}_{n}$ be a bounded sequence of $H_{K}^{s}\left(R^{N}\right)$ for some $s>0$ such that the sequence $\mathbf{u}\left(x+c_{n}\right)$ converges to zero weakly in $H_{K}^{s}\left(R^{N}\right)$ for any sequence $c_{n}$ of elements of $R^{N}$. Then $\mathbf{u}_{n}$ converges to zero strongly in $L_{K}^{p}\left(R^{N}\right)$ for any $p$ in the interval $2<p<\frac{2 N}{N-2 s}(2<p \leq \infty$ if $N<2 s)$.

Proof. Clearly we can take $K=1$.

Let $R>0$ be a fixed positive number and $B_{R}$ the ball centered at the origin and radius $R$. Then, according to Lemma 2.2, Part $6, H^{s}\left(B_{R}\right)=H^{s, 2}\left(B_{R}\right)$ is compactly imbedded in $L^{q}\left(B_{R}\right)$ if

- $2 s \leq N$ and $2 \leq q<\frac{2 N}{N-2 s}$;

- $2 s>N$ and $2 \leq q \leq \infty$.

First we assume $2 s \leq N$. In this case if

$$
2<p<\frac{2 N}{N-2 s}, \quad a=\frac{2}{p} \quad \text { and } \quad q=\max \left(2, \frac{N(p-2)}{2 s}\right)<\frac{2 N}{N-2 s}
$$

then there is constant $c_{1}$ such that:

$$
|u|_{p, \Omega} \leq c_{1}|u|_{s, 2, \Omega}^{a}|u|_{q, \Omega}^{1-a} .
$$

That follows from the interpolation

$$
\left[L^{q}(\Omega), H^{s, 2}(\Omega)\right]_{a}=H^{s^{*}, p^{*}}(\Omega) \quad s^{*}=\text { as } \quad \frac{1}{p^{*}}=\frac{1-a}{q}+\frac{a}{2}
$$

and the imbedding from $H^{s^{*}, p^{*}}(\Omega)$ into $L^{p}(\Omega)$ because due to the choice of $q$ we have

$$
\frac{1}{p^{*}}-\frac{s^{*}}{N}=\frac{1-a}{q}+a\left(\frac{1}{2}-\frac{s}{N}\right) \leq \frac{1}{p}
$$

Next we claim that there is a constant $c_{2}$ such that if $B_{i}$ is a sequence of disjoint balls with radius $R$ of $R^{N}$ then

$$
\sum_{i=1}^{\infty}|u|_{s, 2, B_{i}}^{2} \leq c_{2}|u|_{s, 2, R^{N}}^{2}
$$

In fact, if $X_{i}, i=1,2, \ldots$ and $Y_{i}, i=1,2, \ldots$ are sequences of Hilbert spaces and we define $\ell_{2}\left(X_{1}, X_{2}, \ldots\right)=\{x=$ $\left.\left(x_{1}, x_{2}, \ldots\right): \sum_{i=1}^{\infty}\left|x_{i}\right|^{2}<\infty\right\}$ and $\ell_{2}\left(Y_{1}, Y_{2}, \ldots\right)$ in a similar way, then for $0<a<1$ we have $\left[\ell_{2}\left(X_{1}, X_{2}, \ldots\right)\right.$, $\left.\ell_{2}\left(Y_{1}, Y_{2}, \ldots\right)\right]_{a}=\ell_{2}\left(\left[X_{1}, Y_{1}\right]_{a},\left[X_{2}, Y_{2}\right]_{a}, \ldots\right)$ (see [29], Sect. 1.18.1).

Since clearly (2.6) holds if $s$ is an integer, if we define $X_{i}=L_{2}\left(B_{i}\right)$ and $Y_{i}=H^{m}\left(B_{i}\right)$ where $m \geq s$ is an integer then the map $T u=\left(u_{1}, u_{2}, \cdots\right)$ that takes a function $u$ defined in $R^{N}$ into the sequence of its 
restriction to the ball $B_{i}$ is continuous from $L^{2}\left(R^{N}\right)$ into $\ell_{2}\left(X_{1}, X_{2}, \cdots\right)$ and from $H^{m}\left(R^{N}\right)$ into $\ell_{2}\left(Y_{1}, Y_{2}, \cdots\right)$, and then (2.6) follows by interpolation and this proves the claim. This argument is a slight modification of an argument given in [4].

So, if we take (2.5) with $\Omega=y+B_{R}$, raise it to the power $p$ and we cover the entire space $R^{N}$ by $K(N)$ families of disjoint balls of radius $R$ we see that there is a constant $c_{3}$ such that

$$
\int_{R^{N}}|u(x)|^{p} \mathrm{~d} x \leq c_{3}|u|_{s, 2, R^{N}}^{2} \sup _{y \in R^{N}}\left[\int_{y+B_{R}}|u(x)|^{q}\right]^{\frac{(1-a) p}{q}} .
$$

Finally, for any $p$ and $u_{n}$ satisfying the assumptions of Lemma 2.3, if $q$ is as above, then $(2.7)$ holds with $u=u_{n}$ and since $\sup _{y \in R^{N}}\left[\int_{y+B_{R}}\left|\mathbf{u}_{n}(x)\right|^{q}\right]$ goes to zero because $H^{s}\left(B_{R}\right)$ is compactly imbedded in $L^{q}\left(B_{R}\right)$, Lemma 2.3 is proved in the case $2 s \leq N$.

If $N>2 s$ and $u_{n}$ is as in Lemma 2.3 then due to the compact imbedding from $H^{s}\left(B_{R}\right)$ into $L^{\infty}\left(B_{R}\right)$, we have that $\sup _{y \in R^{N}}\left|u_{n}\right|_{L^{\infty}\left(y+B_{R}\right)}$ tends to zero and this proves Lemma 2.3 in all cases.

Lemma 2.4. Let $\mathbf{u}_{n}$ be a bounded sequence of $H_{K}^{s}\left(R^{N}\right)$ for some $s>0$ such that for some $p$ in the range $2<p<\frac{2 N}{N-2 s}(2<p \leq \infty$ if $N<2 s)$ the sequence $\mathbf{u}_{n}$ is not precompact in $L_{K}^{p}\left(R^{N}\right)$. Then there is a sequence $c_{n} \in R^{N}$ such that some subsequence $\mathbf{u}_{n_{k}}\left(x+c_{n_{k}}\right)$ converges weakly in $H_{K}^{s}\left(R^{N}\right)$ to some $\mathbf{u} \neq 0$ and $\left|c_{n_{k}}\right|$ goes to $\infty$.

Proof. Since for the range of $p^{\prime} s$ we are considering the sequence $\mathbf{u}_{n}$ is precompact in $L_{K}^{p}$ on bounded sets of $R^{N}$, there must be an $\epsilon_{0}>0$ and a sequence $R_{n}$ going to $\infty$ such that $\int_{|x| \geq R_{n}}\left|\mathbf{u}_{n}(x)\right|^{p} \mathrm{~d} x>\epsilon_{0}>0$.

Let $\phi_{n}: R^{N} \rightarrow R$ be a sequence of $C^{\infty}$ functions such that for some integer $m>s$ the derivatives $D^{\alpha} \phi$ of $\phi$ are uniformly bounded on $R^{N}$ if $|\alpha| \leq m, \phi_{n}(x)=0$ if $|x| \leq R_{n}-1$ and $\phi_{n}(x)=1$ if $|x| \geq R_{n}$. Then $\phi_{n} \mathbf{u}_{n}$ is bounded in $H_{K}^{s}\left(R^{N}\right)$ and $\left|\phi_{n} \mathbf{u}_{n}\right|_{p}$ is bounded below by $\epsilon_{0}>0$, Then by the previous lemma, there is a sequence $c_{n} \in R^{N}$ such that some subsequence $\mathbf{u}_{n_{k}}\left(x+c_{n_{k}}\right)$ converges weakly in $H_{K}^{s}\left(R^{N}\right)$ to some $\mathbf{u} \neq 0$. Clearly $\left|c_{n_{k}}\right|$ has to go to $\infty$ and Lemma 2.4 is proved.

Now let $\mathbf{u}(t), t \in\left(-\delta_{0}, \delta_{0}\right)$ be a $C^{2}$ function with values in $H_{K}^{s}\left(R^{N}\right)$ such that

$$
I(\mathbf{u}(t))=\lambda
$$

for any $t \in\left(-\delta_{0}, \delta_{0}\right)$. Such a curve is said to be admissible. Differentiating (2.8) once and twice with respect to $t$ we get

and

$$
I^{\prime}(\mathbf{u}(t)) \dot{\mathbf{u}}(t)=0
$$

where dot means derivative with respect to $t$.

$$
I^{\prime \prime}(\mathbf{u}(t))(\dot{\mathbf{u}}(t), \dot{\mathbf{u}}(t))+I^{\prime}(\mathbf{u}(t)) \ddot{\mathbf{u}}(t)=0,
$$

So, if we denote $\mathbf{u}(0)$ by $\mathbf{u}$, we see that the elements $\dot{\mathbf{h}}$ and $\ddot{\mathbf{h}}$ which are first and second derivatives, respectively, at $t=0$ of an admissible curve must satisfy

$$
I^{\prime}(\mathbf{u}) \dot{\mathbf{h}}=0
$$

and

$$
I^{\prime \prime}(\mathbf{u})(\dot{\mathbf{h}}, \dot{\mathbf{h}})+I^{\prime}(\mathbf{u}) \ddot{\mathbf{h}}=0
$$

We say that a pair $(\dot{\mathbf{h}}, \ddot{\mathbf{h}})$ is admissible for $\mathbf{u}$ if $\dot{\mathbf{h}}$ and $\ddot{\mathbf{h}}$ satisfy the two equations above. The calculation we have performed show that if $\dot{\mathbf{h}}$ and $\ddot{\mathbf{h}}$ are the first and second derivatives, respectively, of an admissible curve, 
then the pair $(\dot{\mathbf{h}}, \ddot{\mathbf{h}})$ is admissible in the sense we have defined. We need the converse with some uniformity with respect to a sequence $\mathbf{u}_{n}$.

Lemma 2.5. Let $\mathbf{u}_{n} \in H_{K}^{s}\left(R^{N}\right)$ be a sequence converging weakly in $H_{K}^{s}\left(R^{N}\right)$ to some $\mathbf{u} \neq 0$ and $\left(\dot{\mathbf{h}}_{n}, \ddot{\mathbf{h}}_{n}\right)$ be a bounded admissible sequence for $\mathbf{u}_{n}$; that is, $\dot{\mathbf{h}}_{n}$ and $\ddot{\mathbf{h}}_{n}$ are bounded sequences in $H_{K}^{s}\left(R^{N}\right)$ satisfying

$$
I^{\prime}\left(\mathbf{u}_{n}\right) \dot{\mathbf{h}_{n}}=0
$$

and

$$
I^{\prime \prime}\left(\mathbf{u}_{n}\right)\left(\dot{\mathbf{h}_{n}}, \dot{\mathbf{h}_{n}}\right)+I^{\prime}\left(\mathbf{u}_{n}\right) \ddot{\mathbf{h}_{n}}=0
$$

Then there are a $\delta_{0}>0$ and a sequence of $C^{2}$ functions $\mathbf{h}_{n}:\left(-\delta_{0}, \delta_{0}\right) \rightarrow H_{K}^{s}\left(R^{N}\right)$ defined for $n$ large and satisfying the following conditions:

i) $\mathbf{h}_{n}(0)=0, \dot{\mathbf{h}}_{n}(0)=\dot{\mathbf{h}}_{n}$ and $\ddot{\mathbf{h}}_{n}(0)=\ddot{\mathbf{h}}_{n}$;

ii) for each $n, \mathbf{u}_{n}+\mathbf{h}_{n}(t)$ is an admissible curve;

iii) $\mathbf{h}_{n}(t), \dot{\mathbf{h}}_{n}(t)$ and $\ddot{\mathbf{h}}_{n}(t)$ are equicontinuous.

Proof. Let $\boldsymbol{\psi}$ be a smooth $R^{K}$-valued function with compact support such that $I^{\prime}(\mathbf{u})(\boldsymbol{\psi}) \neq 0$ and we consider the function

$$
H_{n}(\sigma, t)=I\left(\mathbf{u}_{n}+\sigma \boldsymbol{\psi}+t \dot{\mathbf{h}}_{n}+\frac{t^{2}}{2} \ddot{\mathbf{h}}_{n}\right)-\lambda
$$

Using the Implicit Function theorem at $(0,0)$ we obtain $\sigma_{n}(t)$ such that $H_{n}\left(\sigma_{n}(t), t\right)=0$. Clearly $\mathbf{h}_{n}(t)=$ $\sigma_{n}(t) \boldsymbol{\psi}+t \dot{\mathbf{h}}_{n}+\frac{t^{2}}{2} \ddot{\mathbf{h}}_{n}$ satisfies the conditions i, ii and iii (the fact the we can find a $\delta_{0}>0$ independent of $n$ and the equicontinuity of $\mathbf{h}_{n}(t), \dot{\mathbf{h}}_{n}(t)$ and $\ddot{\mathbf{h}}_{n}(t)$ are a consequence of assumption $\mathbf{H}_{\mathbf{1}}$ about the uniform continuity of the derivatives of $V$ and $I$ on bounded sets) and this proves Lemma 2.5.

The proof of next lemma is elementary and it will not be given.

Lemma 2.6. Let $\mathbf{u}_{n} \in H_{K}^{s}\left(R^{N}\right)$ be a minimizing sequence of problem $(\mathbf{P})$ converging weakly in $H_{K}^{s}\left(R^{N}\right)$ to some $\mathbf{u} \neq 0$. Then:

(i) $\left|V^{\prime}\left(\mathbf{u}_{n}\right)\right| \rightarrow 0$ as $n \rightarrow+\infty$, where $\left|V^{\prime}\left(\mathbf{u}_{n}\right)\right|$ denotes actually the norm of the restriction of the derivative to the admissible hyperplane (that is, the elements $\mathbf{h} \in H_{K}^{s}\left(R^{N}\right)$ such that $I^{\prime}\left(\mathbf{u}_{n}\right) \mathbf{h}=0$ );

(ii) if for some $\delta_{0}>0, \mathbf{h}_{n}:\left(-\delta_{0}, \delta_{0}\right) \rightarrow H_{K}^{s}\left(R^{N}\right)$ is a sequence of $C^{2}$ curves such that $\mathbf{h}_{n}(0)=0, \mathbf{u}_{n}+\mathbf{h}_{n}(t)$ is admissible, $\dot{\mathbf{h}}_{n}(0)$ and $\ddot{\mathbf{h}}_{n}(0)$ are bounded and $\mathbf{h}_{n}(t), \dot{\mathbf{h}}_{n}(t)$ and $\ddot{\mathbf{h}}_{n}(t)$ are equicontinuous then

$$
\liminf \frac{d^{2}}{d t^{2}} V\left(\mathbf{u}_{n}+\mathbf{h}_{n}(t)\right)_{t=0} \geq 0
$$

If $\mathbf{u}$ denotes a generic element of $H_{K}^{s}\left(R^{N}\right)$ such that $I(\mathbf{u})=\lambda$, in order to calculate $\left|V^{\prime}(\mathbf{u})\right|$ on the admissible directions, we have to maximize $V^{\prime}(\mathbf{u}) \mathbf{h}$ for $\mathbf{h} \in H_{K}^{s}\left(R^{N}\right)$ such that $I^{\prime}(\mathbf{u}) \mathbf{h}=0$ and $|\mathbf{h}|_{s}^{2}=1$. Since we are in a Hilbert space and $I^{\prime}(\mathbf{u}) \neq 0$ there is a unique element $\overline{\mathbf{h}}$ where the maximum is achieved and so there are real numbers $\alpha$ and $\gamma$ such that

$$
V^{\prime}(\mathbf{u}) \mathbf{h}+\alpha I^{\prime}(\mathbf{u}) \mathbf{h}+\gamma\left\langle\overline{\mathbf{h}}, \mathbf{h}_{s}\right\rangle=0 \text { for any } \mathbf{h} \in H_{K}^{s}\left(R^{N}\right)
$$

If we set $\mathbf{h}=\overline{\mathbf{h}}$ in (2.14) we see that $\left|V^{\prime}(\mathbf{u})\right|=-\gamma$. 
Moreover, if $\mathbf{h}(t)$ is a smooth curve with $\mathbf{h}(0)=0$ and $\mathbf{u}+\mathbf{h}(t)$ is admissible and we define $W=V+\alpha I$ then

$$
\frac{d^{2}}{d t^{2}} V(\mathbf{u}+\mathbf{h}(t))_{t=0}=W^{\prime \prime}(\mathbf{u})(\dot{\mathbf{h}}, \dot{\mathbf{h}})-\gamma\langle\overline{\mathbf{h}}, \ddot{\mathbf{h}}\rangle_{s}
$$

where $\dot{\mathbf{h}}$ and $\ddot{\mathbf{h}}$ are the first and the second derivative of $\mathbf{h}(t)$ at $t=0$, respectively.

Now let $\mathbf{u}_{n}$ and $\mathbf{u}$ be as in Theorem 2.1. Then from Lemma 2.6 and (2.14) we know that there are sequences of real numbers $\alpha_{n}$ and $\gamma_{n} \rightarrow 0$ and $\overline{\mathbf{h}}_{n} \in H_{K}^{s}\left(R^{N}\right)$ with $\left|\overline{\mathbf{h}}_{n}\right|_{s}=1$ such that

$$
V^{\prime}\left(\mathbf{u}_{n}\right) \mathbf{h}+\alpha_{n} I^{\prime}\left(\mathbf{u}_{n}\right) \mathbf{h}+\gamma_{n}\left\langle\overline{\mathbf{h}}_{n}, \mathbf{h}\right\rangle_{s}=0 \text { for any } \mathbf{h} \in H_{K}^{s}\left(R^{N}\right) .
$$

Lemma 2.7. The sequence $\alpha_{n}$ is bounded.

Proof. If $\left|\alpha_{n}\right|$ tends to infinity for some subsequence (for which we keep the same notation) and we divide (2.16) by $\left|\alpha_{n}\right|$ and we let $n$ to go to infinity we get $I^{\prime}(\mathbf{u})(\mathbf{h})=0$ for any $\mathbf{h} \in H_{K}^{s}\left(R^{N}\right)$, a contradiction in view of assumption $\mathbf{H}_{\mathbf{3}}$ and this proves Lemma 2.7.

Lemma 2.8. The following is true:

i) if $\dot{\mathbf{h}}_{n}$ is a bounded sequence of elements of $H_{K}^{s}\left(R^{N}\right)$ such that $I^{\prime}\left(\mathbf{u}_{n}\right)\left(\dot{\mathbf{h}}_{n}\right)=0$ then

$$
\liminf \left(V^{\prime \prime}\left(\mathbf{u}_{n}\right)\left(\dot{\mathbf{h}}_{n}, \dot{\mathbf{h}}_{n}\right)+\alpha_{n} I^{\prime \prime}\left(\mathbf{u}_{n}\right)\left(\dot{\mathbf{h}}_{n}, \dot{\mathbf{h}}_{n}\right)\right) \geq 0
$$

ii) if $\dot{\mathbf{h}} \in H_{K}^{s}\left(R^{N}\right)$ satisfies $I^{\prime}(\mathbf{u})(\dot{\mathbf{h}})=0$ and $\alpha_{n}$ tends to $\alpha$ then

$$
V^{\prime \prime}(\mathbf{u})(\dot{\mathbf{h}}, \dot{\mathbf{h}})+\alpha I^{\prime \prime}(\mathbf{u})(\dot{\mathbf{h}}, \dot{\mathbf{h}}) \geq 0 .
$$

Proof. Let $\boldsymbol{\psi} \in H_{K}^{s}\left(R^{N}\right)$ be such that $I^{\prime}(\mathbf{u}) \boldsymbol{\psi} \neq 0$ and let us define a sequence of real numbers $d_{n}$ such that $I^{\prime \prime}\left(\mathbf{u}_{n}\right)\left(\dot{\mathbf{h}}_{n}, \dot{\mathbf{h}}_{n}\right)+d_{n} I^{\prime}\left(\mathbf{u}_{n}\right) \boldsymbol{\psi}=0$. The sequence $d_{n}$ is well defined for $n$ large and the pair $\left(\dot{\mathbf{h}}_{n}, d_{n} \boldsymbol{\psi}\right)$ is admissible in the sense of Lemma 2.5. If $\mathbf{h}_{n}(t)$ is the sequence given by that lemma then Part i follows from Lemma 2.6, Part ii, and (2.15) (with $\mathbf{u}=\mathbf{u}_{n}$ and $\mathbf{h}(t)=\mathbf{h}_{n}(t)$ ) because $\gamma_{n}$ tends to zero.

In order to show part ii we have to notice that if $I^{\prime}(\mathbf{u}) \dot{\mathbf{h}}=0$ and we define $\epsilon_{n}$ in such way that $\dot{\mathbf{h}}_{n}=\dot{\mathbf{h}}+\epsilon_{n} \boldsymbol{\psi}$ satisfies $I^{\prime}\left(\mathbf{u}_{n}\right) \dot{\mathbf{h}}_{n}=0$, then Part ii follows from Part i because $\epsilon_{n}$ tends to zero and Lemma 2.8 is proved.

Proof of Theorem 2.1. Argueing by contradiction, suppose that for some $p \in\left(2, \frac{2 N}{N-2 s}\right)(2<p \leq \infty$ if $N<2 s$ ), the sequence $\mathbf{u}_{n}$ is not precompact in $L_{K}^{p}\left(R^{N}\right)$. From Lemma 2.4, the boundedness of $\alpha_{n}$ and passing to a subsequence if necessary, we may assume that $\alpha_{n}$ converges to $\alpha$ and $\mathbf{v}_{n}(x) \hat{=} \mathbf{u}_{n}\left(x+c_{n}\right)$ converges weakly in $H_{K}^{s}\left(R^{N}\right)$ to some $\mathbf{v} \neq 0$, for some sequence $c_{n} \in R^{N}$ such that $\left|c_{n}\right|$ tends to $\infty$.

If we pass to the limit as $n$ tends to $\infty$ in $(2.16)$ we get

$$
V^{\prime}(\mathbf{u})+\alpha I^{\prime}(\mathbf{u})=0
$$

Similarly, if in (2.16) we replace $x$ by $x+c_{n}$ and pass to the limit we get

$$
V^{\prime}(\mathbf{v})+\alpha I^{\prime}(\mathbf{v})=0
$$

Now from assumption $\mathbf{H}_{5}$ we know that there are elements $\mathbf{h}, \mathbf{k} \in H_{K}^{s}\left(R^{N}\right)$ such that

$$
\left(V^{\prime \prime}(\mathbf{u})+\alpha I^{\prime \prime}(\mathbf{u})\right)(\mathbf{h}, \mathbf{h})<0
$$

and

$$
\left(V^{\prime \prime}(\mathbf{v})+\alpha I^{\prime \prime}(\mathbf{v})\right)(\mathbf{k}, \mathbf{k})<0
$$


Next we define $\dot{\mathbf{h}}_{n}=a_{n} \mathbf{h}+b_{n} \mathbf{k}_{n}$ where $\mathbf{k}_{n}(x)=\mathbf{k}\left(x-c_{n}\right)$ and we choose $a_{n}$ and $b_{n}$ in such way that $a_{n}^{2}+b_{n}^{2}=1$ and $I^{\prime}\left(\mathbf{u}_{n}\right)\left(\dot{\mathbf{h}}_{n}\right)=0$. Then

$$
\begin{aligned}
\left(V^{\prime \prime}\left(\mathbf{u}_{n}\right)+\alpha_{n} I^{\prime \prime}\left(\mathbf{u}_{n}\right)\right)\left(\dot{\mathbf{h}}_{n}, \dot{\mathbf{h}}_{n}\right)= & a_{n}^{2}\left(V^{\prime \prime}\left(\mathbf{u}_{n}\right)+\alpha_{n} I^{\prime \prime}\left(\mathbf{u}_{n}\right)\right)(\mathbf{h}, \mathbf{h})+2 a_{n} b_{n}\left(V^{\prime \prime}\left(\mathbf{u}_{n}\right)\right. \\
& \left.+\alpha_{n} I^{\prime \prime}\left(\mathbf{u}_{n}\right)\right)\left(\mathbf{h}, \mathbf{k}_{n}\right)+b_{n}^{2}\left(V^{\prime \prime}\left(\mathbf{v}_{n}\right)+\alpha_{n} I^{\prime \prime}\left(\mathbf{v}_{n}\right)\right)(\mathbf{k}, \mathbf{k})
\end{aligned}
$$

and then and view of assumption $\mathbf{H}_{2}$

$$
\liminf \left(V^{\prime \prime}\left(\mathbf{u}_{n}\right)+\alpha_{n} I^{\prime \prime}\left(\mathbf{u}_{n}\right)\right)\left(\mathbf{h}_{n}, \mathbf{h}_{n}\right)=\liminf a_{n}^{2}\left(V^{\prime \prime}(\mathbf{u})+\alpha I^{\prime \prime}(\mathbf{u})\right)(\mathbf{h}, \mathbf{h})+b_{n}^{2}\left(V^{\prime \prime}(\mathbf{v})+\alpha I^{\prime \prime}(\mathbf{v})\right)(\mathbf{k}, \mathbf{k})<0
$$

a contradiction in view of Lemma 2.8, Part i, and Theorem 2.1 is proved.

Now we formulate some further assumptions that will be used in the next theorem.

$\left.\mathbf{H}_{\mathbf{6}}\right)$ if $\mathbf{u}_{0}$ is an element of $H_{K}^{s}\left(R^{N}\right), \mathbf{v}_{n} \in H_{K}^{s}\left(R^{N}\right)$ is a sequence that converges to zero weakly in $H_{K}^{s}\left(R^{N}\right)$ and $\mathbf{u}_{n}=\mathbf{v}_{n}+\mathbf{u}_{0}$ then $\lim \left(I\left(\mathbf{u}_{n}\right)-I\left(\mathbf{v}_{n}\right)\right)=I\left(\mathbf{u}_{0}\right)$ and $\lim \left(V\left(\mathbf{u}_{n}\right)-V\left(\mathbf{v}_{n}\right)\right)=V\left(\mathbf{u}_{0}\right)$.

$\left.\mathbf{H}_{\mathbf{7}}\right)$ if $\mathbf{u}_{n}$ is bounded sequence in $H_{K}^{s}\left(R^{N}\right)$ converging to $\mathbf{u}$ strongly in $L_{K}^{p}\left(R^{N}\right)$, for any $p$ in the range $2<p<\frac{2 N}{N-2 s}(2<p \leq \infty$ if $N<2 s)$ and $\lim I\left(\mathbf{u}_{n}\right) \neq 0$ then $\lim I^{\prime}\left(\mathbf{u}_{n}\right) \mathbf{u}_{n} \neq 0$

Next theorem will be useful for verifying assumption $\mathbf{H}_{5}$. Results of that type have been proved by Lions in [21]. We give a different proof because we work in a abstract framework and so we cannot use dilation arguments.

Theorem 2.9. Let $\mathbf{u}_{n}$ be a minimizing sequence for problem $(\mathbf{P})$ and suppose $\mathbf{u}_{n}$ converges weakly in $H_{K}^{s}\left(R^{N}\right)$ to some $\mathbf{u}_{0} \neq 0$. Then, under assumptions $\mathbf{H}_{\mathbf{1}}, \mathbf{H}_{\mathbf{3}}, \mathbf{H}_{\mathbf{6}}$ and $\mathbf{H}_{7}, \mathbf{u}_{0}$ is a sub-minimum, that is, if we define $\lambda_{0}=I\left(\mathbf{u}_{0}\right)$ then $\mathbf{u}_{0}$ is a minimizer of problem $(\mathbf{P})$ with $\lambda=\lambda_{0}$.

Proof. Let us define $\mathbf{v}_{n}=\mathbf{u}_{n}-\mathbf{u}_{0}$ and let us consider several cases.

First Case: $\lambda_{0} \neq 0$.

Suppose that there is an element $\mathbf{w} \in H_{K}^{s}\left(R^{N}\right)$ such that $I(\mathbf{w})=\lambda_{0}$ and $V(\mathbf{w})<V\left(\mathbf{u}_{0}\right)$. Then $\mathbf{w} \neq 0$ and so there is an element $\mathbf{h} \in H_{K}^{s}\left(R^{N}\right)$ such that $I^{\prime}(\mathbf{w})(\mathbf{h}) \neq 0$. Since $I\left(\mathbf{w}+\mathbf{v}_{n}\right)$ converges to $\lambda$ (because $I\left(\mathbf{w}+\mathbf{v}_{n}\right)-I(\mathbf{w})-I\left(\mathbf{v}_{n}\right)$ and $I\left(\mathbf{u}_{0}+\mathbf{v}_{n}\right)-I\left(\mathbf{u}_{0}\right)-I\left(\mathbf{v}_{n}\right)$ tend to zero in view of $\mathbf{H}_{6}$ and $\left.I(\mathbf{w})=I\left(\mathbf{u}_{0}\right)\right)$ there is a sequence $t_{n}$ of real numbers tending to zero such that $I\left(\mathbf{w}+t_{n} \mathbf{h}+\mathbf{v}_{n}\right)=\lambda$. Then due to $\mathbf{H}_{\mathbf{1}}$ and $\mathbf{H}_{\mathbf{6}}$ we have $\lim \left(V\left(\mathbf{w}+t_{n} \mathbf{h}+\mathbf{v}_{n}\right)-V\left(\mathbf{u}_{0}+\mathbf{v}_{n}\right)\right)<0$, a contradiction.

Second Case: $\lambda_{0}=0$ and $\mathbf{v}_{n}$ converges to zero strongly in $L_{K}^{p}\left(R^{N}\right)$, for any $p$ in the range $2<p<\frac{2 N}{N-2 s}$ $(2<p \leq \infty$ if $N<2 s)$.

Suppose that there is an element $\mathbf{w} \in H_{K}^{s}\left(R^{N}\right)$ such that $I(\mathbf{w})=0$ and $V(\mathbf{w})<V\left(\mathbf{u}_{0}\right)$. Since $I\left(\mathbf{w}+\mathbf{v}_{n}\right)$ converges to $\lambda$, then in view of assumption $\mathbf{H}_{\mathbf{7}}$, there is a sequence $t_{n}$ converging to one such that $I\left(t_{n}\left(\mathbf{w}+\mathbf{v}_{n}\right)\right)=$ $\lambda$ and then $\lim \left(V\left(t_{n}\left(\mathbf{w}+\mathbf{v}_{n}\right)\right)-V\left(\mathbf{u}_{0}+\mathbf{v}_{n}\right)\right)<0$, a contradiction.

Third Case: $\mathbf{v}_{n}$ does not converge to zero strongly in $L_{K}^{p}\left(R^{N}\right)$, for some $p$ in the range $2<p<\frac{2 N}{N-2 s}$ $(2<p \leq \infty$ if $N<2 s)$.

According to Lemma 2.4, there is a sequence $d_{n} \in R^{N}$ such that $\left|d_{n}\right|$ goes to infinity and $\mathbf{u}\left(x+d_{n}\right)$ converges weakly in $H_{K}^{s}\left(R^{N}\right)$ to some $\mathbf{v} \neq 0$. Let $\mathbf{h}$ be such that $I^{\prime}(\mathbf{v}) \mathbf{h} \neq 0$ and suppose there is an element $\mathbf{w} \in H_{K}^{s}\left(R^{N}\right)$ such that $I(\mathbf{w})=I\left(\mathbf{u}_{0}\right)$ and $V(\mathbf{w})<V\left(\mathbf{u}_{0}\right)$. If we let $\mathbf{h}_{n}(x)=\mathbf{h}\left(x+d_{n}\right)$ there is a sequence of real numbers $t_{n}$ tending to zero such that $I\left(\mathbf{w}+\mathbf{v}_{n}+t_{n} \mathbf{h}_{n}\right)=\lambda\left(\right.$ because $\left.I^{\prime}\left(\mathbf{w}+\mathbf{v}_{n}\right) \mathbf{h}_{n}=I^{\prime}\left(\mathbf{w}_{n}+\mathbf{v}\right) \mathbf{h}\right)$ where $\mathbf{w}_{n}(x)=\mathbf{w}\left(x-d_{n}\right)$ ) and so $\lim \left(V\left(\mathbf{w}+\mathbf{v}_{n}+t_{n} \mathbf{h}_{n}\right)-V\left(\mathbf{u}_{0}+v_{n}\right)\right)<0$, a contradiction, and this proves Theorem 2.9 in all cases.

Next we give a very simple sufficient condition for $\mathbf{H}_{5}$. 
Theorem 2.10. Suppose $V(\mathbf{u})=Q(\mathbf{u})+F(\mathbf{u})$ where $Q(\mathbf{u})$ is quadratic and $Q(\mathbf{u})>0$ for $\mathbf{u} \neq 0$ and let $H(\mathbf{u})=F(\mathbf{u})+\alpha I(\mathbf{u})$. Then assumption $\mathbf{H}_{\mathbf{5}}$ is satisfied provided there is $\beta>1$ such that

$$
H^{\prime \prime}(\mathbf{u})(\mathbf{u}, \mathbf{u}) \leq \beta H^{\prime}(\mathbf{u})(\mathbf{u}) \quad \text { for any } \quad \mathbf{u} \in H_{K}^{s}\left(R^{N}\right)
$$

Proof. Since $Q(\mathbf{u})$ is quadratic, $Q^{\prime \prime}(\mathbf{u})(\mathbf{u}, \mathbf{u})=Q^{\prime}(\mathbf{u})(\mathbf{u})=2 Q(\mathbf{u})$ and then

$$
W^{\prime \prime}(\mathbf{u})(\mathbf{u}, \mathbf{u})=2 Q(\mathbf{u})+H^{\prime \prime}(\mathbf{u})(\mathbf{u}, \mathbf{u}) \leq 2 Q(\mathbf{u})+\beta H^{\prime}(\mathbf{u})(\mathbf{u}) .
$$

Moreover, $V^{\prime}(\mathbf{u})=0$ implies $V^{\prime}(\mathbf{u})(\mathbf{u})=2 Q(\mathbf{u})+H^{\prime}(\mathbf{u})(\mathbf{u})=0$ and then

$$
W^{\prime \prime}(\mathbf{u})(\mathbf{u}, \mathbf{u}) \leq 2(1-\beta) Q(\mathbf{u})<0 \quad \text { if } \mathbf{u} \neq 0
$$

and this proves Theorem 2.10.

As an example, we assume that $F(\mathbf{u})$ and $G(\mathbf{u})$ are sum of homogeneous terms, that is, we suppose $F(\mathbf{u})$ and $I(\mathbf{u})$ can be writen in the form

$$
\begin{aligned}
& F(\mathbf{u})=F_{2}(\mathbf{u})+\sum_{i=1}^{L} F_{p_{i}}(\mathbf{u}) \\
& I(\mathbf{u})=G_{2}(\mathbf{u})+\sum_{j=1}^{M} G_{q_{j}}(\mathbf{u})
\end{aligned}
$$

where $F_{2}$ and $G_{2}$ are homogeneous of degree two and $F_{p_{i}}$ and $G_{q_{j}}$ are homogeneous of degree $p_{i}$ and $q_{j}$, respectively, with

Then (2.17) becomes

$$
2<p_{1}<\cdots<p_{L} \text { and } 2<q_{1}<\cdots<q_{M}
$$

$$
\begin{aligned}
& 2 F_{2}(\mathbf{u})+\sum_{i=1}^{L} p_{i}\left(p_{i}-1\right) F_{p_{i}}(\mathbf{u})+\alpha\left[2 G_{2}(\mathbf{u})+\sum_{j=1}^{K} q_{j}\left(q_{j}-1\right) G_{q_{j}}(\mathbf{u})\right] \\
&\left.\leq \beta\left\{2 F_{2} \mathbf{u}\right)+\sum_{i=1}^{L} p_{i} F_{p_{i}}(\mathbf{u})+\alpha\left[2 G_{2}(\mathbf{u})+\sum_{j=1}^{K} q_{j} G_{q_{j}}(\mathbf{u})\right]\right\}
\end{aligned}
$$

If we know in advance the sign of the multiplier $\alpha$ (and that is possible in some cases) the following result is useful in the applications.

Theorem 2.11. If $\alpha \geq 0$ then assumption $\mathbf{H}_{5}$ is satisfied if either there is an index $i_{0}$ such that

$$
F_{p_{i}}(\mathbf{u}) \geq 0, G_{q_{j}}(\mathbf{u}) \geq 0 \quad \text { for } \quad p_{i}<p_{i_{0}} \quad \text { and } \quad q_{j}<p_{i_{0}}
$$

and

$$
F_{p_{i}}(\mathbf{u}) \leq 0, G_{q_{j}}(\mathbf{u}) \leq 0 \quad \text { for } \quad p_{i}>p_{i_{0}} \quad \text { and } \quad q_{j}>p_{i_{0}}
$$

or there is an index $j_{0}$ such that

$$
F_{p_{i}}(\mathbf{u}) \geq 0, G_{q_{j}}(\mathbf{u}) \geq 0 \quad \text { for } \quad p_{i}<q_{j_{0}} \quad \text { and } \quad q_{j}<q_{j_{0}}
$$

and

$$
F_{p_{i}}(\mathbf{u}) \leq 0, G_{q_{j}}(\mathbf{u}) \leq 0 \quad \text { for } \quad p_{i}>q_{j_{0}} \quad \text { and } \quad q_{j}>q_{j_{0}}
$$


If $\alpha \leq 0$ then assumption $\mathbf{H}_{5}$ is satisfied if $F_{p_{i}}(\mathbf{u}) \geq 0$ for any $i$ and there is an index $j_{0}$ such that $p_{i} \leq q_{j_{0}}$ for any $i$ and $G_{q_{j}}(\mathbf{u}) \geq 0$ for $q_{j}>q_{j_{0}}$ and $G_{q_{j}}(\mathbf{u}) \leq 0$ for $q_{j}<q_{j_{0}}$.

Proof. If $\alpha \geq 0$ condition (2.18) is satisfied with $\beta=p_{i_{0}}-1$ in the first case and $\beta=q_{j_{0}}-1$ in the second.

Similarly, if $\alpha \leq 0$, condition (2.18) is satisfied with $\beta=q_{j_{0}}-1$, and this proves Theorem 2.11.

In the next theorem, either the sign of $\alpha$ is known in advance or it is irrelevant.

Theorem 2.12. Assumption $\mathbf{H}_{5}$ is satisfied if $F_{2}(\mathbf{u})$ is nonnegative, $F_{p_{i}}(\mathbf{u}) \geq 0$ for any $p_{i}, G_{2}(\mathbf{u}) \equiv 0$ and either $G(\mathbf{u}) \equiv G_{q}(\mathbf{u})$ with $p_{i} \leq q$ for any $i$, or $G_{q_{j}}(\mathbf{u}) \geq 0$ for any $j$ and $p_{i} \leq q_{j}$ for any $i$ and $j$.

Proof. If $G(\mathbf{u})=G_{q}(\mathbf{u})$ has a single homogeneous term then condition (2.18) is satisfied with $\beta=q-1$. In the second case, we see that the condition $V^{\prime}(\mathbf{u})(\mathbf{u})+\alpha I^{\prime}(\mathbf{u})(\mathbf{u})=0$ implies $\alpha \leq 0$ and then (2.18) is satisfied if we choose $\beta$ in such way that $p_{i}-1 \leq \beta \leq q_{j}-1$, for any $i$ and $j$, and this proves Theorem 2.12.

If $G(\mathbf{u})=|\mathbf{u}|^{2}$ then we will show that, basically, $\alpha$ is nonnegative and then assumption $\mathbf{H}_{5}$ is satisfied if (2.17) holds with $H=F$. We will come back to this point later in this paper.

We close this section proving a stability result. This type of result has been proved in [10] in case of local functionals and assuming that the constraint is the $L^{2}$ norm. In [26] we have given a proof in the case of a more general functional $V(\mathbf{u})$ but still in the case of a purely quadratic constraint. Since nonquadratic constraints arises in some applications (in the case of BBM equation, for instance) we give here a more general result.

Lemma 2.13. Let $\lambda_{0}>0$ be a real number and let $V$ and $I$ be given functionals on the space $H_{K}^{s}\left(R^{N}\right)$ satisfying assumptions $\mathbf{H}_{\mathbf{1}}, \mathbf{H}_{\mathbf{3}}$ and $\mathbf{H}_{\mathbf{7}}$ and

$\left.\mathbf{H}_{\mathbf{8}}\right)$ there are real numbers $0<\lambda_{1}<\lambda_{0}<\lambda_{2}$ such that for any $B$ there is a $c(B)$ such that if $\lambda_{1} \leq I(\mathbf{u}) \leq$ $\lambda_{2}$ and $V(\mathbf{u}) \leq B$, then $|\mathbf{u}|_{s} \leq c(B)$.

Suppose also that for problem $(\mathbf{P})$ with $\lambda=\lambda_{0}$, minimizing sequences are precompact in $H_{K}^{s}\left(R^{N}\right)$ except for translation in the space variable and denote by $V_{0}$ the minimum of that problem and by $M_{0}$ the set of the admissible elements where the minimum $V_{0}$ is achieved.

If $\mathbf{u}_{n}$ is a sequence of elements of $H_{K}^{s}\left(R^{N}\right)$ such that $I\left(\mathbf{u}_{n}\right)$ tends to $\lambda_{0}$ and $V\left(\mathbf{u}_{n}\right)$ tends to $V_{0}$, then the distance $d\left(\mathbf{u}_{n}, M_{0}\right)$ from $\mathbf{u}_{n}$ to $M_{0}$ tends to zero.

Proof. First we claim that except for translation in the space variable and passing to a subsequence if necessary, we can assume that $\mathbf{u}_{n}$ converges weakly in $H_{K}^{s}\left(R^{N}\right)$ to some $\mathbf{u} \neq 0$. In fact, otherwise, by Lemma $2.3 \mathbf{u}_{n}$ converges to zero strongly in $L_{K}^{p}\left(R^{N}\right)$ for $2<p<\frac{2 N}{N-2 s}(2<p \leq \infty$ if $N<2 s)$ and then according to assumption $\mathbf{H}_{\mathbf{7}}$ we have $\lim I^{\prime}\left(\mathbf{u}_{n}\right) \mathbf{u}_{n} \neq 0$ and then there is sequence $\tau_{n}$ such that $\tau_{n}$ tends to 1 and $I\left(\tau_{n} \mathbf{u}_{n}\right)=\lambda_{0}$. Since $V\left(\tau_{n} \mathbf{u}_{n}\right)$ converges to $V_{0}$ because $V$ is uniformly continuous on bounded sets of $H_{K}^{s}\left(R^{N}\right)$, $\tau_{n} \mathbf{u}_{n}$ is a minimizing sequence for problem $(\mathbf{P})$ with $\lambda=\lambda_{0}$ and this contradicts the fact that minimizing sequences are precompact except for translation and the claim is proved.

If $\mathbf{h} \in H_{K}^{s}\left(R^{N}\right)$ is such that $I^{\prime}(\mathbf{u}) \mathbf{h} \neq 0$ then there is a sequence $t_{n}$ that tends to zero such that $I\left(\mathbf{u}+t_{n} \mathbf{h}+\right.$ $\left.\mathbf{v}_{n}\right)=\lambda_{0}$ where $\mathbf{v}_{n}=\mathbf{u}_{n}-\mathbf{u}$. Since $V\left(\mathbf{u}_{n}\right)-V\left(\mathbf{u}_{n}+t_{n} \mathbf{h}\right)$ tends to zero in view of assumption $\mathbf{H}_{\mathbf{1}}$, we see that $\mathbf{u}_{n}+t_{n} \mathbf{h}$ is a minimizing sequence for problem $(\mathbf{P})$ with $\lambda=\lambda_{0}$ and then $d\left(\mathbf{u}_{n}+t_{n} \mathbf{h}, M_{0}\right)$ tends to zero and this proves Lemma 2.13 .

Under the notation and assumptions of Lemma 2.13 we can prove the following stability result:

Theorem 2.14. Let $\phi(t, \mathbf{u})$ be a map from $R \times H_{K}^{s}\left(R^{N}\right)$ into $H_{K}^{s}\left(R^{N}\right)$ such that $\phi(0, \mathbf{u})=\mathbf{u}, V(\phi(t, \mathbf{u}))=$ $V(\mathbf{u}), I(\phi(t, \mathbf{u}))=I(\mathbf{u})$ and $\phi(t, \mathbf{u})$ commutes with translation in the space variable. Then $M_{0}$ is stable with respect to $\phi(t, \mathbf{u})$ in the following sense: for any $\epsilon>0$ there is $\delta>0$ such that if $d\left(\mathbf{u}, M_{0}\right)<\delta$ then $d\left(\phi(t, \mathbf{u}), M_{0}\right)<\epsilon$ for any $t \in R$.

Proof. Otherwise, there is an $\epsilon_{0}>0$ and a sequences $\mathbf{u}_{n}$ and $t_{n}$ such that $d\left(\mathbf{u}_{n}, M_{0}\right)$ tends to zero and $d\left(\phi\left(t_{n}, \mathbf{u}_{n}\right), M_{0}\right)>\epsilon_{0}$. Then we have:

- $I\left(\phi\left(t_{n}, \mathbf{u}_{n}\right)\right)=I\left(\mathbf{u}_{n}\right)$ tends to $\lambda_{0}$; 
- $V\left(\phi\left(t_{n}, \mathbf{u}_{n}\right)\right)=V\left(\mathbf{u}_{n}\right)$ tends to $V_{0}$

- $d\left(\phi\left(t_{n}, \mathbf{u}_{n}\right), M_{0}\right)>\epsilon_{0}$.

According to Lemma 2.13 this is a contradiction and Theorem 2.14 is proved.

\section{Remarks.}

1) Notice that we have not used continuous dependence of $\phi(t, \mathbf{u})$.

2) If $\phi(t, \mathbf{u})$ is defined for $t \geq 0, V(\phi(t, \mathbf{u})) \leq V(\mathbf{u})$ and $I(\phi(t, \mathbf{u}))=I(\mathbf{u})$, then stability holds for $t \geq 0$.

\section{Application to a CONCREte Class of FUnCtionals}

In this section we consider the concrete problem

$$
\text { (CP) Minimize } V(\mathbf{u}) \text { subject to } I(\mathbf{u})=\lambda>0
$$

where

$$
V(\mathbf{u}) \widehat{=} \frac{1}{2} \int_{R^{N}} m(\xi)|\hat{\mathbf{u}}(\xi)|^{2} \mathrm{~d} \xi+\int_{R^{N}} F(\mathbf{u}(x)) \mathrm{d} x
$$

and

$$
I(\mathbf{u}) \widehat{=} \int_{R^{N}} G(\mathbf{u}(x)) \mathrm{d} x
$$

in the space $H_{K}^{s}\left(R^{N}\right), s \geq 1 / 2$. We denote by $\mathbf{L}$ the selfadjoint operator $\mathbf{L}: D(\mathbf{L}) \subset L_{K}^{2}\left(R^{N}\right) \rightarrow L_{K}^{2}\left(R^{N}\right)$ such that $D(\mathbf{L})=H_{K}^{2 s}\left(R^{N}\right)$ and $\langle\mathbf{L u}, \mathbf{u}\rangle_{L_{K}^{2}\left(R^{N}\right)}=\int_{R^{N}} m(\xi)|\hat{\mathbf{u}}(\xi)|^{2} \mathrm{~d} \xi$ for any $\mathbf{u} \in H_{K}^{2 s}\left(R^{N}\right.$ ) (notice that $\mathbf{L}$ is a diagonal operator, that is, $\left.\mathbf{L}(\mathbf{u})=\left(L\left(u_{1}\right), \cdots, L\left(u_{K}\right)\right)\right)$ and our assumptions are the following:

$\left.\mathbf{H H}_{\mathbf{1}}\right)$ the symbol $m(\xi)$ is a continuous function of $\xi, m(0)=0, m(\xi)>0$ for $\xi \neq 0$ and $\lim _{|\xi| \rightarrow \infty} \frac{m(\xi)}{|\xi|^{2 s}}=A_{1}>0$; $\left.\mathbf{H H}_{\mathbf{2}}\right) F, G: R^{K} \rightarrow R$ are $C^{2}$ satisfying the following conditions:

i) $F(0)=G(0)=0, \operatorname{grad} F(0)=\operatorname{grad} G(0)=0$;

ii) the second derivatives $F^{\prime \prime}(\mathbf{u}), G^{\prime \prime}(\mathbf{u})$ of $F(\mathbf{u})$ and $G(\mathbf{u})$ are Holder continuous in a neighborhood of $\mathbf{u}=0$ and for some $p_{0}<\frac{2 N}{N-2 s}$ (no growth restriction if $N<2 s$ ), and some constant $A_{2}$ and $|\mathbf{u}|$ large, we have

$$
\left|F^{\prime \prime}(\mathbf{u}), G^{\prime \prime}(\mathbf{u})\right| \leq A_{2}|\mathbf{u}|^{p_{0}-2}
$$

$\left.\mathbf{H H}_{3}\right) G\left(\mathbf{u}_{0}\right)>0$ for some $\mathbf{u}_{0} \in R^{K}$ and $I^{\prime}(\mathbf{u}) \neq 0$ for $\mathbf{u} \neq 0$ (a manifold condition);

$\left.\mathbf{H H}_{4}\right) V: H_{K}^{s}\left(R^{N}\right) \rightarrow R$ is bounded below on the admissible set $\left\{\mathbf{u} \in H_{K}^{s}\left(R^{N}\right): I(\mathbf{u})=\lambda\right\}$ and minimizing sequences for problem $\mathbf{C P}$ are bounded in $H_{K}^{s}\left(R^{N}\right)$;

$\left.\mathbf{H H}_{5}\right)$ if $\mathbf{u} \neq 0$ is the weak limit in $H_{K}^{s}\left(R^{N}\right)$ of a minimizing sequence for problem (CP) satisfying the EulerLagrange equation

$$
W^{\prime}(\mathbf{u})=\mathbf{L}(\mathbf{u})+\operatorname{grad} F(\mathbf{u})+\alpha \operatorname{grad} G(\mathbf{u})=0
$$

where $W(\mathbf{u}) \hat{=} V(\mathbf{u})+\alpha I(\mathbf{u})$, then there is an element $\mathbf{h} \in H_{K}^{s}\left(R^{N}\right)$ such that

$$
W^{\prime \prime}(\mathbf{u})(\mathbf{h}, \mathbf{h})=\langle\mathbf{L h}, \mathbf{h}\rangle+\int_{R^{N}} F^{\prime \prime}(\mathbf{u}(x))(\mathbf{h}(x), \mathbf{h}(x)) \mathrm{d} x+\alpha \int_{R^{N}} G^{\prime \prime}(\mathbf{u}(x))(\mathbf{h}(x), \mathbf{h}(x)) \mathrm{d} x<0
$$


$\mathbf{H H}_{6}$ ) (elliptic regularity) if we define $f(\xi)=\frac{\left(1+|\xi|^{2}\right)^{s}}{1+m(\xi)}$ then there is a constant $B$ such that

$$
\int_{\frac{R}{2} \leq|\xi| \leq 2 R}\left|R^{|\alpha|} D_{\alpha} f\right|^{2} \mathrm{~d} \xi / R^{N} \leq B^{2}, \quad 0<R<\infty, \quad|\alpha| \leq \chi
$$

where $\chi$ is the least integer $>N / 2$.

From the assumptions we have made we see that the $\langle\mathbf{L u}, \mathbf{u}\rangle+\int_{R^{N}}|\mathbf{u}(x)|^{2} \mathrm{~d} x$ is a norm equivalent to the norm of $H_{K}^{s}\left(R^{N}\right)$ and the minimization problem is well defined on this space. As an application of our abstract theory we can prove the following:

Theorem 3.1. Under assumptions $\mathbf{H H}_{\mathbf{1}}$ to $\mathbf{H H}_{\mathbf{6}}$, if $\mathbf{u}_{n}$ is a minimizing sequence for problem $(\mathbf{C P})$ and $\mathbf{u}_{n}$ converges weakly in $H_{K}^{s}\left(R^{N}\right)$ to some $\mathbf{u} \neq 0$, then $\mathbf{u}_{n}$ converges to $\mathbf{u}$ strongly in $L_{K}^{p}\left(R^{N}\right), 2<p<\frac{2 N}{N-2 s}$ $(2<p \leq \infty$ if $N<2 s)$ and

$$
\left\langle\mathbf{L}\left(\mathbf{u}_{n}-\mathbf{u}\right),\left(\mathbf{u}_{n}-\mathbf{u}\right)\right\rangle_{L_{K}^{2}\left(R^{N}\right)}=\int_{R^{N}} m(\xi)\left|\hat{\mathbf{u}}_{n}(\xi)-\hat{\mathbf{u}}(\xi)\right|^{2} \mathrm{~d} \xi
$$

tends to zero.

Moreover, there are sequences of real numbers $\alpha_{n}$ and $\gamma_{n}$ and a sequence $\overline{\mathbf{h}}_{n}$ of elements of $H_{K}^{s}\left(R^{N}\right)$ such that

i) $\alpha_{n}$ is bounded and $\gamma_{n}$ tends to zero;

ii) $\left|\overline{\mathbf{h}}_{n}\right|_{s}=1$;

iii) the following approximate Euler-Lagrange equation is satisfied:

$$
\mathbf{L}\left(\mathbf{u}_{n}+\gamma_{n} \overline{\mathbf{h}}_{n}\right)+\operatorname{grad} F\left(\mathbf{u}_{n}(x)\right)+\alpha_{n} \operatorname{grad} G\left(\mathbf{u}_{n}(x)\right)+\gamma_{n} \mathbf{u}_{n}=0 .
$$

Furthermore for some real $\alpha, \mathbf{u}$ satisfies the exact Euler-Lagrange equation

$$
\mathbf{L}(\mathbf{u})(x)+\operatorname{grad} F(\mathbf{u}(x))+\alpha \operatorname{grad} G(\mathbf{u}(x))=0
$$

and $\mathbf{u} \in H_{K}^{2 s+1-\epsilon, r}\left(R^{N}\right), 2 \leq r<\infty, \epsilon>0$.

In particular, $\mathbf{u}$ is $C^{1}$ and together with its first derivatives tends to zero at infinity.

Before proving the Theorem 3.1 we prove a few preliminary results. In all of them we assume that assumptions $\mathbf{H H}_{\mathbf{1}}-\mathbf{H H}_{\mathbf{6}}$ are satisfied.

We start by showing that assumptions $\mathbf{H H}_{\mathbf{1}}$ and $\mathbf{H H}_{\mathbf{2}}$ imply assumptions $\mathbf{H}_{\mathbf{1}}$ and $\mathbf{H}_{\mathbf{2}}$ of Theorem 2.1. The fact that the map that takes $\mathbf{u} \in H_{K}^{s}\left(R^{N}\right)$ into $\int_{R^{N}} F(\mathbf{u}(x)) \mathrm{d} x$ has first and second derivatives follows exactly as in the case of integer Sobolev spaces (see [16]). We will show only that the second derivative is uniformly continuous on bounded sets. The other statements of assumptions $\mathbf{H}_{\mathbf{1}}$ and $\mathbf{H}_{\mathbf{2}}$ follow from imbedding results (Lem. 2.2, Part 5).

Lemma 3.2. Under assumption $\mathbf{H H}_{2}$, the second derivative of the map that takes $\mathbf{u} \in H_{K}^{s}\left(R^{N}\right)$ into $\int_{R^{N}} F(\mathbf{u}(x)) \mathrm{d} x$ is uniformly continuous on bounded sets of $H_{K}^{s}\left(R^{N}\right)$.

Proof. Let $\phi(\mathbf{u})$ be a quadratic form on $R^{K}$ defined for $\mathbf{u} \in R^{K}$ depending continuously on $\mathbf{u}$ and satisfying the growth condition $|\phi(\mathbf{u})| \leq k_{1}+k_{2}|\mathbf{u}|^{r}, r<\frac{4 s}{N-2 s}$ (no growth assumption if $N<2 s$ ) where $k_{1}$ and $k_{2}$ are 
constants. We have to show that for any $C>0$ and any $\epsilon>0$ there is a $\delta>0$ such that if $\mathbf{u}, \mathbf{v}, \mathbf{h}, \mathbf{k} \in H_{K}^{s}\left(R^{N}\right)$, $|\mathbf{u}-\mathbf{v}|_{s}<\delta$ and $|\mathbf{h}|_{s},|\mathbf{k}|_{s} \leq 1$ then

$$
\mid \int_{R^{N}}(\phi(\mathbf{u}(x))-\phi(\mathbf{v}(x))(\mathbf{h}(x), \mathbf{k}(x)) \mathrm{d} x \mid<\epsilon .
$$

Suppose $2 s<N$.

Let us define $A=\left\{x \in R^{N}:|\mathbf{u}(x)| \geq M\right\}$ and $B=\{x:|\mathbf{v}(x)| \geq M\}$, where $M$ will be chosen. Since $\int_{R^{N}}|\mathbf{u}(x)|^{2} \mathrm{~d} x \leq C$, we have meas $(A) \leq \frac{C}{M^{2}}$; similarly, meas $(B) \leq \frac{C}{M^{2}}$. In view of the growth assumption on $\phi(\mathbf{u})$ we have

$$
\left|\int_{A \cup B}(\phi(\mathbf{u}(x))-\phi(\mathbf{v}(x)))(\mathbf{h}(x), \mathbf{k}(x)) \mathrm{d} x\right| \leq \int_{A \cup B}\left(2 k_{1}+k_{2}|\mathbf{u}(x)|^{r}+k_{2}|\mathbf{v}(x)|^{r}\right)|\mathbf{h}(x)||\mathbf{k}(x)| \mathrm{d} x .
$$

Next notice that if a function $g \in L^{q}\left(R^{N}\right)$ for some $q>1$ then $\left|\int_{A} g(x) \mathrm{d} x\right| \leq\left(\operatorname{meas}(A)^{q / q-1}|g|_{L_{q}}\right.$ and then $\int_{A} g(x) \mathrm{d} x$ goes to zero with the measure of the set $A$.

Using Holder's inequality and the imbedding of $H_{K}^{s}\left(R^{N}\right)$ into $L_{K}^{p}\left(R^{N}\right)$ spaces, we can show very easily that for some $q>1$ the $L_{K}^{q}\left(R^{N}\right)$ norm of each integrand in (3.8) is uniformly bounded and then we can find $M$ such that

$$
\left|\int_{A \cup B}(\phi(\mathbf{u}(x))-\phi(\mathbf{v}(x)))(\mathbf{h}(x), \mathbf{k}(x)) \mathrm{d} x\right| \leq \epsilon / 4
$$

Since $\phi(\mathbf{u})$ is uniformly continuous on bounded sets of $R^{K}$ and $|\mathbf{h}(.)|_{L_{K}^{2}\left(R^{N}\right)},|\mathbf{k}(.)|_{L_{K}^{2}\left(R^{N}\right)} \leq 1$ there is a $\delta_{1}>0$ such that

$$
\left|\int_{C}(\phi(\mathbf{u}(x))-\phi(\mathbf{v}(x)))(\mathbf{h}(x), \mathbf{k}(x)) \mathrm{d} x\right| \leq \epsilon / 4
$$

where $C=\left\{x \in R^{N}:|\mathbf{u}(x)-\mathbf{v}(x)| \leq \delta_{1},|\mathbf{u}(x)|,|\mathbf{v}(x)| \leq M\right\}$.

Moreover, if we define $D=\left\{x \in R^{N}:|\mathbf{u}(x)|,|\mathbf{v}(x)| \leq M,|\mathbf{u}(x)-\mathbf{v}(x)|>\delta_{1}\right\}$ then there is a constant $\mathrm{K}$ such that $|\phi(\mathbf{u}(x))-\phi(\mathbf{v}(x))| \leq K|\mathbf{u}(x)-\mathbf{v}(x)|$ for $x \in D$ and then using the imbedding of $H_{K}^{s}\left(R^{N}\right)$ into $L_{K}^{p}\left(R^{N}\right)$ for $2 \leq p \leq \frac{2 N}{N-2 s}$ and Holder's inequality together with $r<\frac{4 s}{N-2 s}$ we conclude that there is $0<\delta_{2} \leq \delta_{1}$ such that if $|\mathbf{u}-\mathbf{v}|_{s}<\delta_{2}$ then

$$
\left|\int_{D}(\phi(\mathbf{u}(x))-\phi(\mathbf{v}(x)))(\mathbf{h}(x), \mathbf{k}(x)) \mathrm{d} x\right|<\epsilon / 4 .
$$

$(3.9,3.10)$ and (3.11) imply (3.7).

The case $N<2 s$ is treated in a similar way and Lemma 3.2 is proved.

\section{Lemma 3.3.}

i) Let $\mathbf{M}: D(\mathbf{M}) \subset X \rightarrow X$ be a selfadjoint operator in a Hilbert space $X$ and suppose there is a continuous linear functional $g$ such that $\langle\mathbf{M h}, \mathbf{h}\rangle \geq 0$ for any $\mathbf{h} \in D(\mathbf{M})$ such that $g(\mathbf{h})=0$. Then the spectrum $\sigma(\mathbf{M})$ cannot have two elements on the negative half line $(-\infty, 0)$;

ii) if an operator of the form $\mathbf{L h}+B \mathbf{h}$ where $\mathbf{L}$ is as above and $B$ is a symmetric $K \times K$ matrix, has no spectrum on the negative half-line then all the eigenvalues of $B$ are nonnegative. 
Proof. Part i is well known (see $[11,21]$ or [24]). In order to prove the second part all we have to do is to notice that the eigenvalue problem $\mathbf{L h}+B \mathbf{h}=\lambda \mathbf{h}$ becomes a diagonal problem if we make the change $\mathbf{h}=P \mathbf{k}$ where $P$ is as orthogonal matrix that diagonalizes $B$ and this proves the lemma because the spectrum of $\mathbf{L}$ is $[0,+\infty)$.

Lemma 3.4. (Elliptic regularity). If $\mathbf{u} \in H_{K}^{s}\left(R^{N}\right)$ solves the Euler-Lagrange equation (3.6) then $\mathbf{u}$ belongs to $H_{K}^{2 s+1-\epsilon, r} 2 \leq r<\infty, \epsilon>0$. In particular $\mathbf{u}$ is $C^{1}$ and together with its first derivatives tends to zero at $\infty$.

Proof. From assumption $\mathbf{H H}_{6}$ and Theorem 2.5 of [15], we know that for any $1<r<\infty$ there is a constant $C(r)$ such that if $\mathbf{h} \in L_{K}^{r}\left(R^{N}\right), \mathbf{u} \in H_{K}^{s}\left(R^{N}\right)$ and $\mathbf{L}(\mathbf{u})+\mathbf{u}=\mathbf{h}$ (this equality is understood in the weak sense) then $\mathbf{u} \in H_{K}^{2 s, r}\left(R^{N}\right)$. If we rewrite (3.6) as

$$
\mathbf{L}(\mathbf{u})(x)+\mathbf{u}(x)=\mathbf{u}(x)-\operatorname{grad} F(\mathbf{u}(x))-\alpha \operatorname{grad} G(\mathbf{u}(x))
$$

then a bootstrap argument shows that if $\mathbf{u}$ solves (3.6) then $\mathbf{u}$ belongs to $H_{K}^{2 s, r} 2 \leq r<\infty$; in particular, $\mathbf{u} \in L_{K}^{\infty}\left(R^{N}\right)$. Since $s \geq 1 / 2$ the right hand side of $(3.12)$ belongs to $W_{K}^{1, r}\left(R^{N}\right), 1<r<\infty$, and from Lemma 2.2, Part 8, we conclude that the right hand side of (3.12) belongs to $H_{K}^{1-\epsilon, r}\left(R^{N}\right)$ for any $\epsilon>0$. If we denote by $\mathbf{k}(x)$ the right hand side of (3.12) we have

$$
\left(1+|\xi|^{2}\right)^{\frac{2 s+1-\epsilon}{2}} \hat{\mathbf{u}}(\xi)=\frac{\left(1+|\xi|^{2}\right)^{s}}{1+m(\xi)}\left(1+|\xi|^{2}\right)^{\frac{1-\epsilon}{2}} \hat{\mathbf{k}}(\xi)
$$

and using $\mathbf{H H}_{6}$ and Theorem 2.5 of [15] again we conclude that $\mathbf{u}$ belongs to $H_{K}^{2 s+1-\epsilon, r} 2 \leq r<\infty, \epsilon>0$ and this proves Lemma 3.4 .

Remark. If $N \leq 2 s$ and $F(\mathbf{u})$ and $G(\mathbf{u})$ have more derivatives, then we can get smoothness of the solutions $\mathbf{u}$ of (3.6) using only the $L_{2}$ theory and then assumption $\mathbf{H H}_{6}$ is not needed (see [2] and [3], for instance).

Lemma 3.5. If $a: R^{N} \rightarrow R$ is continuous and tends to zero as $|x|$ tends to infinity then the operator $S$ : $L_{K}^{2}\left(R^{N}\right) \rightarrow L_{K}^{2}\left(R^{N}\right)$ defined by $S f=(\mathbf{L}+I)^{-1} a()$.$f is compact.$

Proof. It follows immediately from the fact that $S^{*} f=a(.)(\mathbf{L}+I)^{-1} f$ is compact.

Lemma 3.6. Let us write $F(\mathbf{u})$ and $G(\mathbf{u})$ as $F(\mathbf{u})=\frac{1}{2}\langle A \mathbf{u}, \mathbf{u}\rangle+F_{1}(\mathbf{u})$ and $G(\mathbf{u})=\frac{1}{2}\left\langle A_{0} \mathbf{u}, \mathbf{u}\right\rangle+G_{1}(\mathbf{u})$, where $A$ and $A_{0}$ are symmetric $K \times K$ matrices and $F_{1}^{\prime \prime}(0)=G_{1}^{\prime \prime}(0)=0$. If $\mathbf{u} \neq 0$ is the weak limit in $H_{K}^{s}\left(R^{N}\right)$ of a minimizing sequence and $\mathbf{u}$ satisfies the Euler-Lagrange equation (3.6), then all eigenvalues of $A+\alpha A_{0}$ are nonnegative.

Proof. According to Lemma 3.3, Part i, and Lemma 2.8, Part ii, the spectrum of the selfadjoint operator $\mathbf{L}+F^{\prime \prime}(\mathbf{u}(x))+\alpha G^{\prime \prime}(\mathbf{u}(x))$ cannot have two elements on the halfline $(-\infty, 0)$ and since in view of Lemmata 3.4 and $3.5, F_{1}^{\prime \prime}(\mathbf{u}(x))+\alpha G_{1}^{\prime \prime}(\mathbf{u}(x))$ is a compact perturbation of $\mathbf{L}+A+\alpha A_{0}$ and this operator has only continuous spectrum, we see that this operator has the same property and then the conclusion follows from the second part of Lemma 3.3, and this proves Lemma 3.6.

Proof of Theorem 3.1. According to Theorem 2.1 and Lemma 3.2, all is left is to show that $\left\langle\mathbf{L}\left(\mathbf{u}_{n}-\mathbf{u}\right),\left(\mathbf{u}_{n}-\right.\right.$ $\mathbf{u})\rangle_{L_{K}^{2}\left(R^{N}\right)}$ tends to zero.

The first thing to notice is that we can write the aproximate Euler-Lagrange equation in the form (3.5) because when we calculate the norm of $V^{\prime}(\mathbf{u})$ on the admissible elements (Eq. (2.16)), we can take in $H_{K}^{s}\left(R^{N}\right)$ the equivalent norm $\langle\mathbf{L u}, \mathbf{u}\rangle_{L_{K}^{2}\left(R^{N}\right)}+|\mathbf{u}|_{L_{K}^{2}\left(R^{N}\right)}^{2}$. So, passing to a subsequence if necessary, we can assume that $\alpha_{n}$ converges to some $\alpha$. Moreover, the approximate Euler-Lagrange equation (3.5) can be writen as

$$
\mathbf{L}\left(\mathbf{u}_{n}+\gamma_{n} \overline{\mathbf{h}}_{n}\right)+A \mathbf{u}_{n}+\alpha_{n} A_{0} \mathbf{u}_{n}+\operatorname{grad} F_{1}\left(\mathbf{u}_{n}(x)\right)+\alpha_{n} \operatorname{grad} G_{1}\left(\mathbf{u}_{n}(x)\right)+\gamma_{n} \mathbf{u}_{n}=0
$$


and passing to the limit as $n$ tends to infinity we see that $\mathbf{u}$ satisfies the Euler-Lagrange equation

$$
\mathbf{L u}+A \mathbf{u}+\alpha A_{0} \mathbf{u}+\operatorname{grad} F_{1}(\mathbf{u}(x))+\alpha \operatorname{grad} G_{1}(\mathbf{u}(x))=0 .
$$

Using the imbedding of $H_{K}^{s}\left(R^{N}\right)$ in $L_{K}^{r}\left(R^{N}\right)$ for $2 \leq r \leq \frac{2 N}{N-2 s}(2 \leq r<\infty$ if $2 s=N, 2 \leq r \leq \infty$ if $N<2 s)$ the strong convergence of $\mathbf{u}_{n}$ to $\mathbf{u}$ in $L_{K}^{p}\left(R^{N}\right)$ for $2<p<\frac{2 N}{N-2 s}(2<p \leq \infty$ if $N<2 s)$, the assumptions on $F$ and $G$ and Holder's inequality, we see that the integrals

$$
\int_{R^{N}}\left\langle\operatorname{grad} F_{1}\left(\mathbf{u}_{n}(x)\right)-\operatorname{grad} F_{1}(\mathbf{u}(x)), \mathbf{u}_{n}(x)-\mathbf{u}(x)\right\rangle \mathrm{d} x
$$

and

$$
\int_{R^{N}}\left\langle\operatorname{grad} G_{1}\left(\mathbf{u}_{n}(x)\right)-\operatorname{grad} G_{1}(\mathbf{u}(x)), \mathbf{u}_{n}(x)-\mathbf{u}(x)\right\rangle \mathrm{d} x
$$

tend to zero and then, if we subtract (3.14) from (3.13), take the scalar product with $\mathbf{u}_{n}(x)-\mathbf{u}(x)$ and integrate on $R^{N}$ we conclude that

$$
\left\langle\mathbf{L}\left(\mathbf{u}_{n}-\mathbf{u}\right),\left(\mathbf{u}_{n}-\mathbf{u}\right)\right\rangle_{L_{K}^{2}\left(R^{N}\right)}+\int_{R^{N}}\left\langle\left(A+\alpha A_{0}\right)\left(\mathbf{u}_{n}-\mathbf{u}\right),\left(\mathbf{u}_{n}-\mathbf{u}\right)\right\rangle \mathrm{d} x
$$

tends to zero and this proves the theorem because, according to Lemma 3.6, the matrix $A+\alpha A_{0}$ positive.

So, after having proved Theorem 3.1, what is left is

- to give sufficient condtions for $\mathbf{H H}_{\mathbf{3}}, \mathbf{H} \mathbf{H}_{\mathbf{4}}$ and $\mathbf{H H}_{\mathbf{5}}$ (besides the ones given in Ths. 2.10, 2.11 and 2.12);

- to give sufficient conditions to prevent vanishing of minimizing sequences $\mathbf{u}_{n}$, that is, to guarantee that except for translation in the space variable, there is a subsequence (for which we keep the same notation) converging weakly in $H_{K}^{s}\left(R^{N}\right)$ to a nonzero element $\mathbf{u}$; once this is done, by Theorem 3.1 we know that the subsequence $\mathbf{u}_{n}$ converges to $\mathbf{u}$ strongly in $L_{K}^{p}\left(R^{N}\right), 2<p<\frac{2 N}{N-2 s}(2<p \leq \infty$ if $N<2 s)$ and

$$
\int_{R^{N}} m(\xi)\left|\hat{\mathbf{u}_{n}}(\xi)-\hat{\mathbf{u}}(\xi)\right|^{2} \mathrm{~d} \xi
$$

tends to zero;

- to give sufficient conditions to guarantee that the convergence takes place in $L_{K}^{2}\left(R^{N}\right)$ also (this will give the convergence of $\mathbf{u}_{n}$ to $\mathbf{u}$ strongly in $H_{K}^{s}\left(R^{N}\right)$, which is our goal).

Next we discuss assumption $\mathbf{H H}_{3}$. Trivially the manifold condition $\mathbf{H H}_{3}$ is satisfied if $\operatorname{grad} G(\mathbf{u}) \neq 0$ if $\mathbf{u} \neq 0$, but in some cases we can allow $\operatorname{grad} G(\mathbf{u})$ to vanish. For instance, if $s \geq 1$ then the manifold condition is satisfied if $\operatorname{grad} G(\mathbf{u}) \neq 0$ for $\mathbf{u} \neq 0$ and $|\mathbf{u}|$ small. This is a consequence of the following result (see [5], Appendix E.2): if $u \in H^{1}\left(R^{N}\right)$, the sets $\{x: u(x) \leq a\},\{x: u(x) \geq b\}$ have positive measure and $a<b$, then the set $\{x: a<u(x)<b\}$ also has positive measure.

The next result gives a manifold condition in a more delicate case that arises in an application we will make.

Theorem 3.7. If $s=1 / 2, N=1, K=1$ then assumption $\mathbf{H H}_{\mathbf{3}}$ is satisfied if $G^{\prime}(u)$ has a finite number of zeroes.

Theorem 3.7 is a consequence of the next lemmata.

Lemma 3.8. Let $E \subset[a, b]$ be a measurable set contained in the interval $[a, b]$ such that $0<m(E)<b-a$. Then there is a point $c \in[a, b]$ such that

$$
0<\liminf _{h \rightarrow 0} \frac{m(E \cap[c-h, c+h])}{2 h} \leq \limsup _{h \rightarrow 0} \frac{m(E \cap[c-h, c+h])}{2 h}<1
$$


and

$$
0<\liminf _{h \rightarrow 0} \frac{m\left(E^{c} \cap[c-h, c+h]\right)}{2 h} \leq \limsup _{h \rightarrow 0} \frac{m\left(E^{c} \cap[c-h, c+h]\right)}{2 h}<1
$$

In particular

$$
\liminf _{h \rightarrow 0} \frac{m(E \cap[c-h, c+h])}{2 h} \frac{m\left(E^{c} \cap[c-h, c+h]\right)}{2 h}>0 .
$$

Proof. For $x \in[a, b]$ let us define the function

$$
G(x)=m(E \cap[a, x])-\frac{x-a}{b-a} m(E \cap[a, b])
$$

Since $G(a)=G(b)=0$ there is an interior point $c \in(a, b)$ where $G(x)$ achieves either its maximum or its minimum. Let us assume it achieves its maximum at $c$. The other case is treated similarly. Since

$$
\limsup _{h \rightarrow 0_{+}} \frac{G(c+h)-G(c)}{h} \leq 0 \quad \text { and } \quad \liminf _{h \rightarrow 0_{+}} \frac{G(c)-G(c-h)}{h} \geq 0
$$

we conclude that

and

$$
\limsup _{h \rightarrow 0_{+}} \frac{m(E \cap[c, c+h])}{h} \leq \frac{m(E \cap[a, b])}{b-a}
$$

and then

$$
\liminf _{h \rightarrow 0_{+}} \frac{m(E \cap[c, c+h])}{h} \geq \frac{m(E \cap[a, b])}{b-a}
$$

$$
\frac{m(E \cap[a, b])}{2} \leq \liminf _{h \rightarrow 0} \frac{m(E \cap[c-h, c+h])}{2 h} \leq \limsup _{h \rightarrow 0} \frac{m(E \cap[c-h, c+h])}{2 h}<\frac{1+m(E \cap[a, b])}{2}
$$

and this proves (3.16). (3.17) follows from (3.16) and this proves Lemma 3.8.

Lemma 3.9. Let $E_{1}, \cdots E_{m}$ be a finite number of disjoint measurable sets of $R$ such that $0<m\left(E_{i}\right)<+\infty$, $i=1, \cdots, m$ and let $a_{1}<a_{2} \cdots<a_{m}$ be a sequence of nonzero real numbers. If we denote by $I_{i}$ the caracteristic function of the set $E_{i}$, then the function $u(x)=\sum_{i=1}^{m} a_{i} I_{i}(x)$ does not belong to the space $H^{1 / 2}(R)$.

Proof. According Lemma 2.2, Part 1, we have to show that the integral

$$
\int_{R^{2}} \frac{|u(x)-u(y)|^{2}}{|x-y|^{2}} \mathrm{~d} x \mathrm{~d} y
$$

diverges and so it suffices to show, for instance, that the integral

$$
\int_{E_{1} \times E_{1}^{c}} \frac{|u(x)-u(y)|^{2}}{|x-y|^{2}} \mathrm{~d} x \mathrm{~d} y
$$

diverges. Since outside $E_{1}$ the function $u(x)$ assumes a finite number of values none of them being equal to $a_{1}$, all we have to do is to show that the integral

$$
\int_{E_{1} \times E_{1}^{c}} \frac{1}{|x-y|^{2}} \mathrm{~d} x \mathrm{~d} y
$$


diverges. Let us assume by contradiction that it converges and let $c$ the number given by Lemma 3.8 . Then we must have

$$
\lim _{h \rightarrow 0_{+}} \int_{\left(E_{1} \cap[c-h, c+h]\right) \times\left(E_{1}^{c} \cap[c-h, c+h]\right)} \frac{1}{|x-y|^{2}} \mathrm{~d} x \mathrm{~d} y=0 .
$$

However, that integral is bounded below by $m(E \cap[c-h, c+h]) m\left(E_{1} \cap[c-h, c+h]\right) / h^{2}$ and then this limit has to be zero and this is a contradiction in view of Lemma 3.8 and Lemma 3.9 is proved.

Notice that the characteristic function of a bounded interval belongs to $H^{s}(R)$ for $s<1 / 2$ and so Theorem 3.7 deals with a critical case.

Now we discuss how to prevent vanishing of a minimizing sequences as well as its convergence in $L_{K}^{2}\left(R^{N}\right)$. The final argument of the proof Theorem 3.1 together with (3.15) shows that if the matrix $A+\alpha A_{0}$ is positive definite, then the convergence takes place in $L_{K}^{2}\left(R^{N}\right)$ also. In order to give sufficient conditions for $A+\alpha A_{0}$ to be positive definite, we have to consider two cases according to the form of the quadratic part of $F(\mathbf{u})$ and $G(\mathbf{u})$. In each case we also give a sufficient condition for $\mathbf{H H}_{\mathbf{3}}$.

We use the decomposition $F(\mathbf{u})=\frac{1}{2}\langle A \mathbf{u}, \mathbf{u}\rangle+F_{1}(\mathbf{u})$ and $G(\mathbf{u})=\frac{1}{2}\left\langle A_{0} \mathbf{u}, \mathbf{u}\right\rangle+G_{1}(\mathbf{u})$ as in Lemma 3.6.

First case: $A_{0}$ is positive definite.

In that case, following [25], in view of the constraint we can replace $F(\mathbf{u})$ by $F(\mathbf{u})+\beta G(\mathbf{u})$ and the quadratic part of the new $F(\mathbf{u})$ will be $A+\beta A_{0}$. Then, choosing $\beta$ properly, we can assume that the following normalization condition is satisfied:

(NC) the symmetric matrix $A$ has at least one zero eigenvalue and all the others are nonnegative.

Theorem 3.10. If $G_{1}(\mathbf{u}) \geq 0$ and

$$
\lim _{|\mathbf{u}| \rightarrow+\infty} \frac{F_{-}(\mathbf{u})}{|\mathbf{u}|^{\gamma}}=0
$$

where $\gamma=2+\frac{4 s}{N}$ and $F_{-}(\mathbf{u})$ denotes the negative part of $F(\mathbf{u})$ then assumption $\mathbf{H H}_{4}$ is satisfied.

Proof. The proof follows very closely the proof given in [20], Part II, for $s=1$. Using the interpolation

$$
\left[L_{K}^{2}\left(R^{N}\right), H_{K}^{s, 2}\right]_{a}=H_{K}^{a s, 2} \quad a=2 / \gamma
$$

and the imbedding of $H_{K}^{a s, 2}\left(R^{N}\right)$ into $L_{K}^{\gamma}\left(R^{N}\right)$ because $\frac{1}{\gamma}=\frac{1}{2}-\frac{a s}{N}$ we see that there is a constant $c_{7}$ such that

$$
|\mathbf{u}|_{\gamma, R^{N}}^{\gamma} \leq c_{7}|\mathbf{u}|_{s, 2, R^{N}}^{2}|\mathbf{u}|_{2, R^{N}}^{(1-a) \gamma}
$$

and this proves Theorem 3.10 because we have an a priori bound for the $L_{K}^{2}\left(R^{N}\right)$ norm of $\mathbf{u}_{n}$.

Lemma 3.11. Under the normalization condition (NC), any minimizing sequence $\mathbf{u}_{n}$ of problem (CP) has a subsequence converging weakly (modulo translation in the space variable) in $H_{K}^{s}\left(R^{N}\right)$ to a nonzero element $\mathbf{u} \in H_{K}^{s}\left(R^{N}\right)$ if and only $V(\lambda)<0$, where $V(\lambda)$ is the infimum of $V$ on the admissible set.

Proof. If $\mathbf{w} \in R^{K}$ is an eigenvector of $A$ associated to the zero eigenvalue, let $\phi: R^{N} \rightarrow R$ be a smooth function such that $\mathbf{v}(x)=\phi(x) \mathbf{w}$ is admissible. It is easy to see that there is a $c(\sigma)$ that tends to 1 as $\sigma$ tends to zero such that $\mathbf{v}_{\sigma}(x)=c(\sigma) \sigma^{N / 2} \mathbf{v}(\sigma x)$ is also admissible. Moreover

$$
V\left(\mathbf{v}_{\sigma}\right)=\frac{c^{2}(\sigma)}{2} \int_{R^{N}} m(\sigma \xi)|\hat{\mathbf{v}}(\xi)|^{2} \mathrm{~d} \xi+\sigma^{-N} \int_{R^{N}} F_{1}\left(\sigma^{N / 2} \mathbf{v}(x)\right) \mathrm{d} x
$$


where $F_{1}$ is as in Lemma 3.6 because $A \mathbf{w}=0$. Due to Lebesgue theorem, the first term of last equality tends to zero (because $m(0)=0$ ). Furthermore, since the sup norm of $\sigma^{N / 2} \mathbf{v}(x)$ tends to zero as $\sigma$ tends to zero and $F_{1}(\mathbf{v})$ is of order higher than two at $\mathbf{v}=0$, the second term also tends to zero and then $V\left(\mathbf{v}_{\sigma}\right)$ tends to zero as $\sigma$ tends to zero.

Conversely, suppose $V(\lambda)<0$ and, argueing by contradiction, suppose there is a bounded minimizing sequence $\mathbf{u}_{n}$ such that $\mathbf{u}_{n}\left(\cdot+c_{n}\right)$ tends weakly to zero in $H_{K}^{s}\left(R^{N}\right)$ for any sequence $c_{n}$. Then according to Lemma $2.3, \mathbf{u}_{n}$ converges to zero strongly in $L_{K}^{p}\left(R^{N}\right), 2<p<\frac{2 N}{N-2 s}(2<p \leq \infty$ if $N<2 s)$ and then $\liminf V\left(\mathbf{u}_{n}\right) \geq 0$ (because the quadratic part of $V(\mathbf{u})$ is nonnegative in view of the normalization condition), a contradiction and this proves the lemma.

Next we give two sufficient conditions for $V(\lambda)$ being negative.

Theorem 3.12. $V(\lambda)<0$ if either

- there is a $\mathbf{u}_{0} \in R^{K}$ such that $F\left(\mathbf{u}_{0}\right)<0, G\left(\mathbf{u}_{0}\right)>0$ and $\lambda$ is large, or

- $\lim _{\sigma \rightarrow 0_{+}} V\left(\mathbf{u}_{\sigma}\right)=-\infty$ where $\gamma_{0}=2+\frac{4 s_{0}}{N}$ and $s_{0}>0$ is the largest number such that $\frac{|m(\xi)|}{|\xi|^{2 s_{0}}}$ remains bounded as $|\xi|$ goes to zero.

Proof. In the first case, if $\mathbf{u}$ is a smooth function with compact support such that $\int_{R^{N}} F(\mathbf{u}(x)) \mathrm{d} x<0$ and $\int_{R^{N}} G(\mathbf{u}(x)) \mathrm{d} x>0$ and we define $\mathbf{u}_{\sigma}(x)=\mathbf{u}(\sigma x)$ then

$$
V\left(\mathbf{u}_{\sigma}\right)=\frac{1}{2 \sigma^{n}} \int_{R^{N}} m(\sigma \xi)|\hat{\mathbf{u}}(\xi)|^{2} \mathrm{~d} \xi+\frac{1}{\sigma^{n}} \int_{R^{N}} F(\mathbf{u}(x)) \mathrm{d} x .
$$

Moreover, for any $\epsilon>0$ there is a $K(\epsilon)$ such that $m(\xi) \leq \epsilon+K(\epsilon)|\xi|^{2 s}$ and then

$$
V\left(\mathbf{u}_{\sigma}\right) \leq \frac{1}{2 \sigma^{n}} \int_{R^{N}}\left(\epsilon+K(\epsilon) \sigma^{2 s}|\xi|^{2 s}\right)\left(|\hat{\mathbf{u}}(\xi)|^{2} \mathrm{~d} \xi+\frac{1}{\sigma^{n}} \int_{R^{N}} F(\mathbf{u}(x)) \mathrm{d} x .\right.
$$

If we fix $\epsilon$ small in such way that

$$
\frac{\epsilon}{2} \int_{R^{N}}|\hat{\mathbf{u}}(\xi)|^{2} \mathrm{~d} \xi+\int_{R^{N}} F(\mathbf{u}(x)) \mathrm{d} x<0
$$

then $\lim _{\sigma \rightarrow 0_{+}} V\left(\mathbf{u}_{\sigma}\right)=-\infty($ because $s>0)$ and $I\left(\mathbf{u}_{\sigma}\right)=\frac{1}{\sigma^{N}} I(\mathbf{u})$ tends to $+\infty$.

In the second case, let $\mathbf{u} \in H_{K}^{s}\left(R^{N}\right)$ be such that $\hat{\mathbf{u}}(\xi)$ has compact support $A$ and $\int_{R^{N}}\langle A \mathbf{u}(x), \mathbf{u}(x)\rangle \mathrm{d} x=\lambda$.

Then there is $c(\sigma)$ tending to 1 as $\sigma$ tends to zero such that $\mathbf{u}_{\sigma}(x) \widehat{=} c(\sigma) \sigma^{N / 2} \mathbf{u}(\sigma x)$ admissible.

Moreover, since $\mathbf{u}(x)$ is bounded, if $B$ is an arbitrarily large positive number, then for $\sigma$ small enough we have $F\left(\sigma^{N / 2} \mathbf{u}(x)\right) \leq-B \sigma^{\gamma N / 2}|\mathbf{u}(x)|^{\gamma}$ and then for some constant $K_{1}$ we have

$$
V\left(\mathbf{u}_{\sigma}\right) \leq K_{1} \sigma^{2 s_{0}} \int_{A}|\xi|^{2 s_{0}}|\hat{\mathbf{u}}(\xi)|^{2} \mathrm{~d} \xi-B \sigma^{-N} \int_{R^{N}} \sigma^{\gamma N / 2}|\mathbf{u}(x)|^{\gamma} \mathrm{d} x
$$

and this expression is negative if $\sigma$ is small enough and this proves Theorem 3.12.

In order to discuss the convergence in the $L^{2}$ norm we make a further assumption $\mathbf{H H}_{\mathbf{7}}$. Let us recall that we are assuming the normalization condition (NC) is satisfied. 
$\mathbf{H H}_{\mathbf{7}}$ ) If $\mathbf{u}$ is a nontrivial solution of the Euler equation

$$
L \mathbf{u}+\operatorname{grad} F(\mathbf{u})=0
$$

then $V(\mathbf{u}) \geq 0$

Theorem 3.13. Under assumptions $\mathbf{H H}_{\mathbf{1}}-\mathbf{H H}_{\mathbf{6}}$, the Normalization Condition (NC) and assumption $\mathbf{H H}_{\mathbf{7}}$ any minimizing sequence of problem $(\mathbf{C P})$ is precompact in $H_{K}^{s}\left(R^{N}\right)$ except for translation if and only if the infimum $V(\lambda)$ of $V$ on the admissible set is negative. Moreover any minimizer satisfies the Euler-Lagrange equation (3.6) with $\alpha>0$.

Proof. As we have seen, under (NC) there is a sequence $\mathbf{u}_{n}$ of admissible functions whose $L_{K}^{\infty}\left(R^{N}\right)$ tends to zero and $V\left(\mathbf{u}_{n}\right)$ tends to zero. So, the condition $V(\lambda)<0$ is necessary for precompactness of minimizing sequences except for translation.

If $V(\lambda)<0$ then according to Theorems 3.1 and 3.11, if $\mathbf{u}_{n}$ is a minimizing sequence for problem $(\mathbf{C P})$ then except for translation and passing to a subsequence for which we keep the same notation, $\mathbf{u}_{n}$ converges to some $\mathbf{u} \neq 0$ strongly in $L_{K}^{p}\left(R^{N}\right)$ for $2<p<\frac{2 N}{N-2 s}(2<p \leq \infty$ if $2 s>N), \int_{R^{N}} m(\xi)\left|\hat{\mathbf{u}_{n}}(\xi)-\hat{\mathbf{u}}(\xi)\right|^{2} \mathrm{~d} \xi$ tends to zero and $\mathbf{u}$ satisfies the Euler-Lagrange equation (3.6). Moreover, the normalization condition (NC) implies that the quadratic part of the functional $V$ is convex and then we must have $V(\mathbf{u}) \leq 0$. We also have $\alpha \geq 0$ because $A+\alpha A_{0}$ is positive according to Lemma 3.6 and $A$ has zero as an eigenvalue. From assumption $\mathbf{H H}_{\mathbf{7}}$ we conclude that $\alpha>0$ and then, in view of (3.15), this implies convergence in the $L_{K}^{2}\left(R^{N}\right)$ norm because the matrix $A+\alpha A_{0}$ is positive definite and this proves Theorem 3.13.

Next we give two sufficient conditions of $\mathbf{H H}_{\mathbf{7}}$.

Theorem 3.14. Assumption $\mathbf{H H}_{\mathbf{7}}$ is satisfied if either

i) there is a number $\beta>2$ such that $\langle\operatorname{grad} F(\mathbf{u}), \mathbf{u}\rangle \leq \beta F(\mathbf{u})$

or

ii) $\left\langle m^{\prime}(\xi), \xi\right\rangle>0$ for $\xi \neq 0$.

Proof. In the first case if we take the scalar product of (3.20) with $\mathbf{u}$ and integrate we have $\int_{R^{N}}\langle L \mathbf{u}(x), \mathbf{u}(x)\rangle+$ $\left.\int_{R^{N}} \operatorname{grad} F(\mathbf{u}(x)), \mathbf{u}(x)\right\rangle \mathrm{d} x=0$ and then

$$
\begin{aligned}
V(\mathbf{u}) & \left.=\frac{1}{2} \int_{R^{N}}\langle L \mathbf{u}(x), \mathbf{u}(x)\rangle \mathrm{d} x+\int_{R^{N}} F(\mathbf{u}(x)) \mathrm{d} x \geq \frac{1}{2} \int_{R^{N}}\langle L \mathbf{u}(x), \mathbf{u}(x)\rangle \mathrm{d} x+\frac{1}{\beta} \int_{R^{N}} F(\mathbf{u}(x)), \mathbf{u}(x)\right\rangle \mathrm{d} x \\
& \geq\left(\frac{1}{2}-\frac{1}{\beta}\right) \int_{R^{N}}\langle L \mathbf{u}(x), \mathbf{u}(x)\rangle>0
\end{aligned}
$$

In the second case, as we will see later in this paper (Eq. (3.21)), for solutions of (3.20) we have

$$
V(\mathbf{u})=\frac{1}{2 N} \int_{R^{N}}\langle\operatorname{grad} m(\xi), \xi\rangle|\hat{\mathbf{u}}(\xi)|^{2} \mathrm{~d} \xi
$$

and this proves Theorem 3.14.

Remark. Notice that condition assumption i of Theorem 3.13 is compatible with condition (2.17) of Theorem 2.10.

Second case: $A_{0}=0$ and $A_{1}$ is positive definite.

Theorem 3.15. Under assumptions $\mathbf{H H}_{\mathbf{1}}-\mathbf{H H}_{\mathbf{6}}$ and except for translation, any minimizing sequence is precompact in $H_{K}^{s}\left(R^{N}\right)$. 
Proof. If $\mathbf{u}_{n}$ is a minimizing sequence and $\mathbf{u}_{n}$ converges to zero strongly in in $L_{K}^{p}\left(R^{N}\right), 2<p<\frac{2 N}{N-2 s}$ $(2<p \leq \infty$ if $N<2 s)$ then the constraint is violated for large $n$. Then, in view of Lemma 2.3 and except for translation in the $x$ variable, we can assume that $\mathbf{u}_{n}$ converges weakly in $H_{K}^{s}\left(R^{N}\right)$ to a nonzero element $\mathbf{u}$ and so, according to Theorem 3.1 all is left is to prove the convergence of $\mathbf{u}_{n}$ in $L_{K}^{2}\left(R^{N}\right)$ but this follows immediately from (3.15) because $A_{0}=0$ and $A_{1}$ is positive definite and this proves Theorem 3.15.

In order to give other sufficient conditions for $\mathbf{H H}_{5}$ (besides the one given by Ths. 2.10, 2.11 and 2.12) we start by recalling a result known as Derrick's theorem [13] that says the following: if $N \geq 3$ and $\mathbf{u} \in H_{K}^{1}\left(R^{N}\right)$ is a nontrivial critical point of the functional

$$
W(\mathbf{u})=\frac{1}{2} \int_{R^{N}}|\operatorname{grad} \mathbf{u}(x)|^{2} \mathrm{~d} x+\int_{R^{N}} H(\mathbf{u}(x)) \mathrm{d} x,
$$

then under appropriate smoothness and growth assumptions on $H(\mathbf{u})$, there is an element $\mathbf{h} \in H_{K}^{1}\left(R^{N}\right)$ such that

$$
W^{\prime \prime}(\mathbf{u})(\mathbf{h}, \mathbf{h})=\int_{R^{N}}|\operatorname{grad} \mathbf{h}(x)|^{2} \mathrm{~d} x+\int_{R^{N}} H^{\prime \prime}(\mathbf{u}(x))(\mathbf{h}(x), \mathbf{h}(x)) \mathrm{d} x<0 .
$$

The argument is the following: if we define the curve $\mathbf{u}_{k}(x)=\mathbf{u}(x / k)$ then

$$
W(k)=W\left(\mathbf{u}_{k}\right)=\frac{k^{N-2}}{2} \int_{R^{N}}|\operatorname{grad} \mathbf{u}(x)|^{2} \mathrm{~d} x+k^{N} \int_{R^{N}} H(\mathbf{u}(x)) \mathrm{d} x .
$$

Moreover, since $\mathbf{u}$ is a critical point of $W$ we must have

$$
W^{\prime}(1)=\frac{(N-2)}{2} \int_{R^{N}}|\operatorname{grad} \mathbf{u}(x)|^{2} \mathrm{~d} x+N \int_{R^{N}} H(\mathbf{u}(x)) \mathrm{d} x=0
$$

(which is the so called Pohozaev identity) and then

$$
W^{\prime \prime}(1)=\frac{(N-2)(N-3)}{2} \int_{R^{N}}|\operatorname{grad} \mathbf{u}(x)|^{2} \mathrm{~d} x+N(N-1) \int_{R^{N}} H(\mathbf{u}(x)) \mathrm{d} x=(2-N) \int_{R^{N}}|\operatorname{grad} \mathbf{u}(x)|^{2} \mathrm{~d} x<0
$$

and this shows that $W^{\prime \prime}(\mathbf{u})(\mathbf{h}, \mathbf{h})<0$ where $\mathbf{h}(x)=\sum_{i=1}^{N} x_{i} \frac{\partial \mathbf{h}(x)}{\partial x_{i}}$.

Of course we have to be carefull because, in general, we do not know if the function $\mathbf{h}(x)=\sum_{i=1}^{N} x_{i} \frac{\partial \mathbf{h}(x)}{\partial x_{i}}$ is an element of $H_{K}^{1}\left(R^{N}\right)$. That can be fixed by constructing a sequence $\mathbf{h}_{n}(x)=\phi_{n}(x) \mathbf{h}(x)$, where $\phi_{n}(x)$ is a sequence of truncation, so that $W^{\prime \prime}(\mathbf{u})\left(\mathbf{h}_{n}, \mathbf{h}_{n}\right)<0$ if $n$ is large.

If $N=2$ we can also prove that statement by perturbing and truncating $\mathbf{h}(x)=\sum_{i=1}^{N} x_{i} \frac{\partial \mathbf{h}(x)}{\partial x_{i}}(\operatorname{see}[25])$.

All this together means that if we are dealing with functionals

$$
V(\mathbf{u})=\frac{1}{2} \int_{R^{N}}|\operatorname{grad} \mathbf{u}(x)|^{2} \mathrm{~d} x+\int_{R^{N}} F(\mathbf{u}(x)) \mathrm{d} x, \quad I(\mathbf{u})=\int_{R^{N}} G(\mathbf{u}(x) \mathrm{d} x
$$

and $N \geq 2$ then assumption $\mathbf{H H}_{5}$ is always satisfied and that is independent of assuming that the critical point $\mathbf{u}$ is the weak limit of a minimizing sequence. 
The same argument works for functionals of the type

$$
V(\mathbf{u})=\frac{1}{2} \int_{R^{3}}(\operatorname{div} \mathbf{E}(x))^{2} \mathrm{~d} x+\gamma \int_{R^{3}}|\operatorname{curl} \mathbf{E}(x)|^{2} \mathrm{~d} x+\int_{R^{N}} F(\mathbf{E}(x)) \mathrm{d} x \quad \gamma>0 .
$$

It works also in the presence of a nonlocal term $\int_{R^{N} \times R^{N}} \frac{F(u(x)) F(u(y))}{|x-y|^{\sigma}} \mathrm{d} x \mathrm{~d} y$ with homogeneous kernel.

If $N=1$ then the argument above fails but if we restrict ourselves to critical points which are weak limits of minimizing sequences (and this is all we need to consider), then we can show that assumtpion $\mathbf{H H}_{5}$ is also satisfied for that type of functionals. The reason is that such critical points are sub-minima according to Theorem 2.9 and due to a result of symmetry of minimizers, they will be even functions of $x$ and this allows us to prove what we need (see [25]).

So, for functionals of the type

$$
V(\mathbf{u})=\frac{1}{2} \int_{R^{N}}|\operatorname{grad} \mathbf{u}(x)|^{2} \mathrm{~d} x+\int_{R^{N}} F(\mathbf{u}(x)) \mathrm{d} x, \quad I(\mathbf{u})=\int_{R^{N}} G(\mathbf{u}(x) \mathrm{d} x
$$

assumption $\mathbf{H H}_{5}$ is always satisfied (notice that we are dealing with vector value functions $\mathbf{u}(x)$ ) and then we can show the existence of minimizer for very general nonlinearities $F(\mathbf{u})$ and $G(\mathbf{u})$ (see [25]).

In order to try to use use Derrick's argument in the case of the functional

$$
W(\mathbf{u})=\frac{1}{2} \int_{R^{N}} m(\xi)|\hat{\mathbf{u}}(\xi)|^{2} \mathrm{~d} \xi+\int_{R^{N}} H(\mathbf{u}(x)) \mathrm{d} x
$$

we define $\mathbf{u}_{k}(x)=\mathbf{u}(x / k)$ and since $\widehat{\mathbf{u}_{k}(x)}=k^{N} \hat{\mathbf{u}}(k \xi)$, we have

$$
W(k)=W\left(\mathbf{u}_{k}\right)=\frac{k^{N}}{2} \int_{R^{N}} m(\xi / k)|\hat{u}(\xi)|^{2} \mathrm{~d} \xi+k^{N} \int_{R^{N}} H(\mathbf{u}(x)) \mathrm{d} x
$$

and then

and

$$
W^{\prime}(1)=\frac{N}{2} \int_{R^{N}} m(\xi)|\hat{\mathbf{u}}(\xi)|^{2} \mathrm{~d} \xi-\frac{1}{2} \int_{R^{N}}\left\langle m^{\prime}(\xi), \xi\right\rangle|\hat{\mathbf{u}}(\xi)|^{2} \mathrm{~d} \xi+N \int_{R^{N}} H(\mathbf{u}(x)) \mathrm{d} x
$$

$$
\begin{aligned}
W^{\prime \prime}(1)= & \frac{N(N-1)}{2} \int_{R^{N}} m(\xi)|\hat{\mathbf{u}}(\xi)|^{2} \mathrm{~d} \xi+(1-N) \int_{R^{N}}\left\langle m^{\prime}(\xi), \xi\right\rangle|\hat{\mathbf{u}}(\xi)|^{2} \mathrm{~d} \xi \\
& +\frac{1}{2} \int_{R^{N}}\left\langle m^{\prime \prime}(\xi) \xi, \xi\right\rangle|\hat{\mathbf{u}}(\xi)|^{2} \mathrm{~d} \xi+N(N-1) \int_{R^{N}} H(\mathbf{u}(x)) \mathrm{d} x
\end{aligned}
$$

So, if $\mathbf{u}$ is a critical point of $W$ we have $W^{\prime}(1)=0$,

$$
W(\mathbf{u})=\frac{1}{2 N} \int_{R^{N}}\left\langle m^{\prime}(\xi), \xi\right\rangle|\hat{\mathbf{u}}(\xi)|^{2} \mathrm{~d} \xi
$$

and

$$
W^{\prime \prime}(1)=\frac{1}{2}\left[-(N-1) \int_{R^{N}}\left\langle m^{\prime}(\xi), \xi\right\rangle|\hat{\mathbf{u}}(\xi)|^{2} \mathrm{~d} \xi+\int_{R^{N}}\left\langle m^{\prime \prime}(\xi) \xi, \xi\right\rangle|\hat{\mathbf{u}}(\xi)|^{2} \mathrm{~d} \xi\right]
$$

and so $W^{\prime \prime}(1)<0$ if we assume that

$$
-(N-1)\left\langle m^{\prime}(\xi), \xi\right\rangle+\left\langle m^{\prime \prime}(\xi) \xi, \xi\right\rangle<0 \quad \text { for } \xi \neq 0
$$


Notice that if $N=1$ and, for instance, $K=1$, then (3.22) imposes that $m^{\prime \prime}(\xi) \leq 0$ and this is not compatible with the other assumptions we have made on $m(\xi)$. So, as in the case of local integrals, in the case of space dimension $N=1$, Derrick's argument can never be used to show that assumption $\mathbf{H H}_{5}$ is satisfied.

Fortunately, at least in the case $K=1$ (that is, $u(x)$ is real valued), if a maximum principle is available, then assumption $\mathbf{H H}_{5}$ is satisfied. For instance, if $u \in H^{2}\left(R^{N}\right)$ is a nontrivial solution of the equation

$$
-\Delta u(x)+h(u(x))=0
$$

then $v(x)=\frac{\partial u(x)}{\partial x_{i}}$ satisfies

$$
-\Delta v(x)+h^{\prime}(u(x)) v(x)=0
$$

and this means that $v(x)$ is an eigenfunction associated to the zero eigenvalue of the linear operator $-\Delta+h^{\prime}(u(x))$ and, since $v(x)$ changes sign, it cannot be the principal eigenfunction. So, there is a negative eigenvalue and this gives another proof for $K=1$ that assumption $\mathbf{H H}_{5}$ is satisfied for $V(u)=1 / 2 \int_{R^{N}}|\operatorname{grad} u(x)|^{2} \mathrm{~d} x+$ $\int_{R^{N}} F(u(x)) \mathrm{d} x$ and $I(u)=\int_{R^{N}} G(u(x) \mathrm{d} x$.

For functionals $V$ of the form

$$
V(u)=\frac{1}{2} \int_{R^{N}}|\operatorname{grad} u(x)|^{2} \mathrm{~d} x+\int_{R^{N}} F_{1}(u(x)) \mathrm{d} x-\int_{R^{N} \times R^{N}} k(x-y) F_{2}(u(x)) F_{2}(u(y)) \mathrm{d} x \mathrm{~d} y
$$

then we can show that a maximum principle holds (and hence, assumption $\mathbf{H H}_{5}$ is satisfied) if $k(z) \leq 0$ (the atractive case) and the derivative $f_{2}(u)$ of $F_{2}(u)$ satisfied $f_{2}(u) \geq 0$ [23]. If $k(z)$ is radial and nonincreasing, symmetrization can be used [19].

In the class of problems we are dealing with here and under some assumptions on the multiplier $m(\xi)$, a maximum principle can be proved and, as a consequence of it, assumption $\mathbf{H H}_{\mathbf{5}}$ can be verified for a very large class of nonlinearities. This will be done next.

Theorem 3.16. Suppose $K=1(u(x)$ is scalar) and that for $\mu$ large we have $K(x)>0$, where $K(x)$ is defined by $\hat{K}(\xi)=\frac{1}{\mu+m(\xi)}$. Then assumptions $\mathbf{H H}_{5}$ is satisfied.

Proof. In fact, from Lemma 3.4 we see that we can differentiate (3.3) with respect to, say, $x_{1}$ and then we conclude that zero is an eigenvalue of corresponding to an eigenfunction that changes sign. Moreover, according to Theorem 2.9 and Lemma 3.6, the essential spectrum of the operator $\mathbf{L}+F^{\prime \prime}(\mathbf{u}(x))+\alpha G^{\prime \prime}(\mathbf{u}(x))$ is a half-line $[C,+\infty)$ where $C \geq 0$. Then the proof given in [3] for Proposition 2 (p. 355) applies and Theorem 3.16 is proved.

\section{Applications to stability of waves}

In this last section we show how we can combine Theorems 2.14, 3.12-3.14 and 3.15 to show the existence and the stability of a set of solitary waves for some differential and integro-differential equations.

First example. We consider generalized intermediate long-wave equation

$$
u_{t}+\left(f(u(x))_{x}-\beta_{1} M_{1} u_{x}-\beta_{2} M_{2} u_{x}=0\right.
$$

where $\beta_{i}>0, i=1,2, M_{i}$ is the Fourier multiplier defined by

$$
\left(\widehat{M}_{i} w\right)(\xi)=m_{i}(\xi) \hat{w}(\xi) \quad m_{i}(\xi)=\xi \operatorname{coth}\left(\xi H_{i}\right)-\frac{1}{H_{i}}
$$


for $i=1,2, H_{i}$ a real number. Equation (4.1) has the following two first integrals:

$$
V(u)=\frac{1}{2} \int_{R} m(\xi)|\hat{u}(\xi)|^{2} \mathrm{~d} \xi+\int_{R} F(u(x)) \mathrm{d} x \quad I(u)=\int_{R} u^{2}(x) \mathrm{d} x
$$

where $m(\xi)=\beta_{1} m_{1}(\xi)+\beta_{2} m_{2}(\xi), F^{\prime}(u)=f(u)$ and $F(0)=0$. In [4] it has been proved that assumption $\mathbf{H H}_{\mathbf{1}}$ is satisfied for $s=1 / 2$ and that the assumption $K(x)>0$ of Theorem 3.16 is also satisfied. An elementary calculation shows that condition ii of Theorem 3.14 is also satisfied. All this together with Theorems 3.12 and 3.13 allow us to conclude that if $F(u)$ satisfies the normalization condition $F^{\prime \prime}(0)=0$ and we want to minimize $V(u)$ under $I(u)=\lambda>0$, then minimizing sequences are precompact in $H^{1 / 2}(R)$ except for translation if

$$
\lim _{|u| \rightarrow+\infty} \frac{F_{-}(u)}{|u|^{4}}=0
$$

where $F_{-}(u)$ denotes the negative part of $F(u)$, and

- either

i) $\lim _{|u| \rightarrow 0} \frac{F(u)}{|u|^{6}}=-\infty$ (and $\lambda$ is any positive number)

or

ii) $F\left(u_{0}\right)<0$ for some $u_{0}$ and $\lambda>0$ and large.

If we define $J(u)=\langle L u, u\rangle_{s}$, then $J(|u|) \leq J(u)$ (see [4]) and then making an even extension of $F(u)$ we can show the existence of positive minimizers. The symmetry of the minimizer can also be proved using a lemma of Riesz (see [4]).

For the generalized BO we have $m(\xi)=|\xi|$ and the conclusion is the same as above because the condition $K(x)>0$ of Theorem 3.16 is satisfied (see [3], p. 364), and condition ii of Theorem 3.14 is also satisfied (the lack of differentiability of $m(\xi)$ at $\xi=0$ does not cause serious problems).

Second example. We consider the generalized KdV:

$$
u_{t}-u_{x x x}+(f(u))_{x}=0
$$

The functionals $V(u)=\int_{R}\left(\frac{u_{x}^{2}(x)}{2}+F(u(x)) \mathrm{d} x\right.$ and $I(u)=\int_{R} u^{2}(x) \mathrm{d} x$ are first integrals of (4.2) and if the normalization condition $F^{\prime \prime}(0)=0$ is satisfied and we want to minimize $V(u)$ under $I(u)=\lambda>0$ in the space $H^{1}(R)$ then according to theory we have presented, minimizing sequences are precompact in $H^{1}(R)$ except for translation if

$$
\lim _{|u| \rightarrow+\infty} \frac{F_{-}(u)}{|u|^{6}}=0
$$

where $F_{-}(u)$ denotes the negative part of $F(u)$, and

- either

i) $\lim _{|u| \rightarrow 0} \frac{F(u)}{|u|^{6}}=-\infty$ (and $\lambda$ is any positive number)

or

ii) $F\left(u_{0}\right)<0$ for some $u_{0}$ and $\lambda>0$ and large.

In the next two examples we study BBM type equations

$$
u_{t}-L u_{t}+(f(u))_{x}=0
$$


where $L$ is given by $\widehat{L u}(\xi)=m(\xi) \hat{u}(\xi)$. Equation (4.3) has the following two first integrals

$$
V(u)=\int_{R}\left(L u(x) u(x)+u^{2}(x)\right) \mathrm{d} x, \quad I(u)=\int_{R} F(u(x)) \mathrm{d} x
$$

where $F(0)=0$ and $F^{\prime}(u)=f(u)$. We assume that $m(\xi)$ satisfies the assumption $\mathbf{H H}_{\mathbf{1}}$ and we consider the problem of minimizing $V(u)$ under $I(u)=\lambda>0$ in the space $H^{s}(R)$. Clearly, $V$ is bounded below and minimizing sequences are bounded in $H^{s}(R)$. We also assume that the number of zeroes of $f(u)$ is finite (so that, according to Th. 3.7, the manifold condition is satisfied if $s=1 / 2)$ and we make the decomposition $F(u)=u^{2}+F_{1}(u)$ as in Lemma 3.6 and then the normalized functional (for which we keep the same notation) is $V(u)=\int_{R}\left(L u(x) u(x)-F_{1}(u(x)) \mathrm{d} x\right.$. In order to be more specific we consider two separate cases.

Third example. In (4.3) we take $m(\xi)$ as in the first example.

If we want to minimize $V(u)$ under $I(u)=\lambda>0$, then according to the theory we have presented, minimizing sequences are precompact in $H^{1 / 2}(R)$ if

- either

i) $\lim _{|u| \rightarrow 0} \frac{F_{1}(u)}{|u|^{6}}=+\infty$ (and $\lambda$ is any positive number)

- or

ii) $F_{1}\left(u_{0}\right)>0$ for some $u_{0}$ and $\lambda>0$ and large.

Fourth example. In (4.3) we take $L(u)=-u_{x x}$. In this case if we want to minimize $V(u)$ under $I(u)=\lambda>0$, then minimizing sequences are precompact in $H^{1}(R)$ if

- either

i) $\lim _{|u| \rightarrow 0} \frac{F_{1}(u)}{|u|^{6}}=\infty$ (and $\lambda$ is any positive number)

- or

ii) $F_{1}\left(u_{0}\right)>0$ for some $u_{0}$ and $\lambda>0$ and large.

In this last case, if $f(u)=|u|^{p}$ with $p>6$ and $\lambda>0$ is small, then the infimum of the normalized $V(u)$ under $I(u)=\lambda$ is equal to zero and it is not achieved (because using interpolation inequalities we see that $V(u)>0$ if $u$ is admissible and $\lambda$ is small).

It is easy to see that the equation $-u_{x x}+c u+(c-1) u|u|^{p-2}=0$ for critical points of the constrained variational problem has a nontrivial solution $u_{c}(x)$ for any $0<c<1$ but, if $c<1$ is close to one then this solution cannot be global minimizers of the variational problem above because $I\left(u_{c}\right)$ tends to zero as $c$ tends to 1. This fact is consistent with a conjecture of Weinstein [31] according to which for $p>6$ the traveling waves of the BBM equation with small speed are unstable.

Notice that if $F(u)$ has no quadratic term and $f(u)$ has a finite number of zeroes, then according to Theorem 3.15, then for any level $\lambda \neq 0$, there is a stable set of traveling waves.

We finish this paper indicating further applications of our method. In [25] we have shown the existence of minimizer for the problem

under

$$
V(u, v)=\int_{R}\left(\frac{1}{2} u_{x}^{2}+\frac{1}{2} v_{x}^{2}+F(u(x), v(x))\right) \mathrm{d} x
$$

$$
\int_{R}\left(u^{2}(x)+v^{2}(x)\right) \mathrm{d} x=\lambda
$$


for very general nonlinearities $F(u, v)$ and this gives us existence and stability of traveling waves for the KdV system

$$
\begin{aligned}
& u_{t}=-u_{x x x}+\frac{\mathrm{d}}{\mathrm{d} x} F_{u}(u, v) \\
& v_{t}=-v_{x x x}+\frac{\mathrm{d}}{\mathrm{d} x} F_{v}(u, v) .
\end{aligned}
$$

In [25] we have also made an application to a Schrodinger system and a Klein-Gordon equation. As a further application, we can minimize the energy of the system:

$$
i \frac{\partial \mathbf{E}}{\partial t}+\operatorname{curl} \operatorname{curl} \mathbf{E}+\gamma \operatorname{grad} \operatorname{div} \mathbf{E}+f\left(|\mathbf{E}|^{2}\right) \mathbf{E}=0
$$

for a given value of the charge $\int_{R^{N}}|\mathbf{E}|^{2} \mathrm{~d} x$. For certain nonlinearities it has been proved that the minimizer is not the gradient of a radial function [12].

The Zakharov-Kuznetsov equation and its BBM version [8] have first integrals of the type we have considered and so our theory can be used to show the existence and the stability of a set of traveling waves for those equations.

\section{REFERENCES}

[1] R. Adams, Sobolev Spaces. Academic Press (1975).

[2] J. Albert, Concentration-Compactness and stability-wave solutions to nonlocal equations. Contemp. Math. 221, AMS (1999) $1-30$.

[3] J. Albert, J. Bona and D. Henry, Sufficient conditions for stability of solitary-wave solutions of model equations for long waves. Phys. D 24 (1987) 343-366.

[4] J. Albert, J. Bona and J.C. Saut, Model equations for waves in stratified fluids. Proc. Roy. Soc. London Ser. A 453 (1997) $1233-1260$.

[5] J. Bergh and J. Lofstrom, Interpolation Spaces. Springer-Verlag, New-York/Berlin (1976).

[6] P. Blanchard and E. Bruning, Variational Methods in Mathematical Physics. Springer-Verlag (1992).

[7] H. Brezis and E. Lieb, Minimum Action Solutions of Some Vector Field Equations. Comm. Math. Phys. 96 (1984) 97-113.

[8] A. de Bouard, Stability and instability of some nonlinear dispersive solitary waves in higher dimension. Proc. Roy. Soc. Edinburgh Sect. A 126 (1996) 89-112.

[9] I. Catto and P.L. Lions, Binding of atoms and stability of molecules in Hartree and Thomas-Fermi type theories, Part I. Comm. Partial Differential Equations 17 (1992) 1051-1110.

[10] T. Cazenave and P.L. Lions, Orbital Stability of Standing waves for Some Nonlinear Schrödinger Equations. Comm. Math. Phys. 85 (1982) 549-561.

[11] S. Coleman, V. Glazer and A. Martin, Action Minima among to a class of Euclidean Scalar Field Equations. Comm. Math. Phys. 58 (1978) 211-221.

[12] T. Colin and M. Weinstein, On the ground states of vector nonlinear Schrödinger equations. Ann. Inst. H. Poincaré Phys. Théor. 65 (1996) 57-79.

[13] G.H. Derrick, Comments on Nonlinear Wave Equations as Models for Elementary Particles. J. Math. Phys. 5, 9 (1964) $1252-1254$.

[14] M. Grillakis, J. Shatah and W. Strauss, Stability of Solitary Waves in the Presence of Symmetry I. J. Funct. Anal. 74 (1987) 160-197.

[15] L. Hormander, Estimates for translation invariant operators in $L^{p}$ spaces. Acta Math. 104 (1960) 93-140.

[16] O. Kavian, Introduction à la théorie des points critiques et applications aux problèmes elliptiques. Springer, Heidelberg (1993).

[17] P. Lax, Integrals of nonlinear equations of evolution and solitary waves. Comm. Pure Appl. Math. 21 (1968) 467-490.

[18] S. Levandosky, Stability and instability of fourth-order solitary waves. J. Dynam. Differential Equations 10 (1998) 151-188.

[19] E. Lieb, Existence and uniqueness of minimizing solutions of Choquard's nonlinear equation. Stud. Appl. Math. 57 (1977) 93-105.

[20] P.L. Lions, The Concentration-Compactness Principle in the Calculus of Variations. Ann. Inst. H. Poincaré Anal. Non Linéaire 1 (1984) Part I 109-145, Part II 223-283.

[21] P.L. Lions, Solutions of Hartree-Fock Equations for Coulomb Systems. Comm. Math. Phys. 109 (1987) 33-97. 
[22] O. Lopes, Radial symmetry of minimizers for some translation and rotation invariant functionals. J. Differential Equations 124 (1996) 378-388.

[23] O. Lopes, Sufficient conditions for minima of some translation invariant functionals. Differential Integral Equations 10 (1997) 231-244.

[24] O. Lopes, A Constrained Minimization Problem with Integrals on the Entire Space. Bol. Soc. Brasil Mat. (N.S.) 25 (1994) $77-92$.

[25] O. Lopes, Variational Systems Defined by Improper Integrals, edited by L. Magalhaes et al., International Conference on Differential Equations. World Scientific (1998) 137-153.

[26] O. Lopes, Variational problems defined by integrals on the entire space and periodic coefficients. Comm. Appl. Nonlinear Anal. 5 (1998) 87-120.

[27] J. Maddocks and R. Sachs, On the stability of KdV multi-solitons. Comm. Pure. Appl. Math. 46 (1993) 867-902.

[28] J.C. Saut, Sur quelques généralizations de l'équation de Korteweg-de Vries. J. Math. Pure Appl. (9) 58 (1979) $21-61$.

[29] H. Triebel, Interpolation Theory, Functions Spaces, Differential Operators. North-Holland, Amsterdam (1978).

[30] M. Weinstein, Liapunov Stability of Ground States of Nonlinear Dispersive Evolution Equations. Comm. Pure Appl. Math. 39 (1986) 51-68.

[31] M. Weinstein, Existence and dynamic stability of solitary wave solution of equations arising in long wave propagation. Comm. Partial Differential Equations 12 (1987) 1133-1173. 